\title{
Maximal real Schottky groups
}

Rubén A. Hidalgo

\begin{abstract}
Let $S$ be a real closed Riemann surfaces together a reflection $\tau: S \rightarrow S$, that is, an anticonformal involution with fixed points. A well known fact due to C.L. May [19] asserts that the group $K(S, \tau)$, consisting on all automorphisms (conformal and anticonformal) of $S$ which commutes with $\tau$, has order at most 24(g-1). The surface $S$ is called maximally symmetric Riemann surface if $|K(S, \tau)|=24(g-1)[8]$. In this note we proceed to construct real Schottky uniformizations of all maximally symmetric Riemann surfaces of genus $g \leq 5$. A method due to Burnside [3] permits us the computation of a basis of holomorphic one forms in terms of these real Schottky groups and, in particular, to compute a Riemann period matrix for them. We also use this in genus 2 and 3 to compute an algebraic curve representing the uniformized surface $S$. The arguments used in this note can be programed into a computer program in order to obtain numerical approximation of Riemann period matrices and algebraic curves for the uniformized surface $S$ in terms of the parameters defining the real Schottky groups.
\end{abstract}

\section{Introduction}

Classical uniformization theory asserts that each closed Riemann surface of genus $g \geq 2$ can be uniformized by (i) Fuchsian groups acting on the hyperbolic plane $\mathbb{H}$; (ii) Schottky groups and (iii) described by projective algebraic curves. To give explicit relations between these objects is very difficult. In case the Riemann surface $S$ has a group $H$ of conformal automorphisms so that $S / H$ is the sphere with three branch values (a rigid surface), it is not hard to find an explicit Fuchsian group and an algebraic curve representing $S$. In [4] P. Buser and R. Silhol have considered the case of hyperelliptic

2000 Mathematics Subject Classification: 30F10, 30F40, 30F50.

Keywords: Schottky groups, Riemann surfaces, Riemann matrices. 
Riemann surfaces with a reflection (an anticonformal involution with fixed points). They are able to obtain an algebraic curve starting from the Fuchsian group computing the capacity of certain hyperbolic polygons. Another results in this direction are given, for instance, in M. Seppälä [22] and GianniSeppälä-Silhol-Trager [7]. In general, the explicit computation of Riemann matrices is hard and it is related to the famous Schottky problem: To give a characterization of the Riemann matrices of closed Riemann surfaces inside the space of symmetric matrices with positive imaginary part. A method due to Burnside [3] permits the computation of a basis of the holomorphic one forms for certain real Schottky groups and, in particular, to compute Riemann period matrices and algebraic curves of closed Riemann surfaces admitting a reflection. This is the approach we consider in this note for maximally symmetric Riemann surfaces of genus $g \leq 5$. In particular, we are able to give a transcendental equation on a parameter $p \in(2-\sqrt{3}, 1)$ which defines a Schottky uniformization of the closed Riemann surface defined by the algebraic curve $y^{2}=x^{6}-1$.

\section{Real Schottky groups}

Let $g$ be some positive integer. Assume we have a collection of $2 g$ pairwise disjoint simple loops, say $\alpha_{1}, \ldots, \alpha_{g}, \widetilde{\alpha}_{1}, \ldots, \widetilde{\alpha}_{g}$, bounding a common region $\mathcal{D}$ of connectivity $2 g$. Let us also assume we have a collection of Möbius transformations $A_{1}, \ldots, A_{g}$ (necessarily loxodromic ones) so that $A_{j}\left(\alpha_{j}\right)=\widetilde{\alpha}_{j}$ and $A_{j}(\mathcal{D}) \cap \mathcal{D}=\emptyset$, for all $j=1, \ldots, g$. The group $G$ generated by $A_{1}, \ldots, A_{g}$ turns out to be a Kleinian group, purely loxodromic, isomorphic to a free group of rank $g$ and with connected region of discontinuity [15], called a Schottky group of genus $g$ (for another equivalent definitions of Schottky groups one can see, for instance, [17] and [5]). The domain $\mathcal{D}$ is called a standard fundamental domain and the loops $\alpha_{1}, \ldots, \alpha_{g}, \widetilde{\alpha}_{1}, \ldots, \widetilde{\alpha}_{g}$ a fundamental set of loops for $G$. If we denote by $\Omega$ the region of discontinuity of $G$, then $\Omega / G$ is a closed Riemann surface of genus $g$. Retrosection theorem [1] states that if $S$ is a closed Riemann surface of genus $g$, then there is a Schottky group $G$ (necessarily of genus $g$ ) with region of discontinuity $\Omega$ and $P: \Omega \rightarrow S$ a normal covering map, with $G$ as covering group. We say that $(G, \Omega, P: \Omega \rightarrow X)$ is a Schottky uniformization of $S$. A simple proof of this fact was also given by L. Bers in [1] using quasiconformal mappings. In the case we can choose the above loops $\alpha_{j}$ and $\widetilde{\alpha}_{j}$ (for all $j$ ) as Euclidean circles, for some set of free generators of the Schottky group, we say that $G$ is a classical Schottky group. The existence of non-classical Schottky groups is well known [14]. A Schottky group $G$, of genus $g$, is called hyperelliptic if there is a Möbius transformation $C$ of order two such that $C G C^{-1}=G$ 
and the group generated by $G$ and $C$ uniformizes an sphere (necessarily with $2(g+1)$ branched points of order two). Equivalently, there is a set of free generators $A_{1}, \ldots, A_{g}$ for $G$ so that $C A_{j} C=A_{j}^{-1}$ for $j=1, \ldots, g$. The hyperelliptic Schottky groups uniformize hyperelliptic Riemann surfaces and, reciprocally, every hyperelliptic Riemann surface can be uniformized by such a group [13]. In a similar manner, there are certain Schottky groups which permit the uniformization of real surfaces (surfaces with a reflection). A real Schottky group is by definition a Schottky group $G$ which keeps invariant a circle $L$ in the Riemann sphere; equivalently, there is reflection $\sigma: \widehat{\mathbb{C}} \rightarrow \widehat{\mathbb{C}}$ (with $L$ as its set of fixed points) which commutes with every element of $G$. It is not hard to see that a real Schottky group is classical for every set of generators. If $G$ is a real Schottky group, with associated reflection $\sigma$, then the uniformized Riemann surface $S=\Omega(G) / G$ admits a reflection $\tau: S \rightarrow S$, which is induced by $\sigma$. Reciprocally, each Riemann surface $S$ admitting a reflection $\tau$ can be constructed in such a way.

Theorem 2.1 ([16]) Let $S$ be a closed Riemann surface admitting a reflection $\tau: S \rightarrow S$. Then there is a real Schottky group, with associated reflection $\sigma$, uniformizing $S$ so that $\tau$ is induced by $\sigma$.

A simple argument of this fact is as follows. Let us consider the quotient Klein surface (with non-empty border) $S / \tau$. Assume that the surface $S / \tau$ is orientable. In this case, $S / \tau$ is a closed Riemann surface of genus $\gamma$ with $k>0$ components in its border ( $k$ is the number of components of fixed points of $\tau$ ). The genus of $S$ is $g=2 \gamma+k-1$. We may uniformize this surface $S / \tau$ by a group $K$, generated by the reflection $\sigma$ on the unit circle and a Fuchsian group (acting in the unit disc) uniformizing a Riemann surface of genus $\gamma$ and $k$ holes. This is consequence of quasiconformal deformation theory and the fact that reflections has circles as set of fixed points. The index two subgroup of orientation preserving transformations (the above Fuchsian group) is then a real Schottky group, with associated reflection $\sigma$, uniformizing $S$ as desired. Now, assume the surface $S / \tau$ is non-orientable. In this case, $S / \tau$ is the connected sum of $\gamma>0$ projective real planes and $k>0$ boundary components. The genus of $S$ is $g=\gamma+k-1$. We may uniformize this surface $S / \tau$ by a group $K$, generated by the reflection $\sigma$ on the unit circle and a Fuchsian group (acting in the unit disc and containing glidereflections) uniformizing a non-orientable Klein surface of genus $\gamma$ and $k$ holes. This is again consequence of quasiconformal deformation theory and the fact that reflections has circles as set of fixed points. The index two subgroup of orientation preserving transformations is then a real Schottky group, with associated reflection $\sigma$, uniformizing $S$ as desired. 
Remark. The above result asserts then every closed Riemann surface admitting a reflection can be uniformized by a classical Schottky group. An open problem is to decide if every closed Riemann surface can be uniformized by a classical Schottky group.

Given a closed Riemann surface $S$ and a group $H$ of automorphisms (conformal or anticonformal ones), we may wonder for the existence of a Schottky uniformization of $S$ for which the group $H$ can be lifted. If it is possible to find such a Schottky uniformization, then we say that $H$ is of Schottky type. Necessary and sufficient conditions for a group $H$ to be of Schottky type are given in [9], [10], [11] and [12] for the case that $H$ only contains conformal automorphisms. In the case there are anticonformal automorphisms the above result asserts the following two facts.

Corollary 2.2. Let $S$ be a closed Riemann surface admitting a reflection $\tau: S \rightarrow S$. Let us denote by $K(S, \tau)$ the group of conformal and anticonformal automorphisms of $S$ commuting with $\tau$. Then the group $K(S, \tau)$ is of Schottky type.

Proof. This is clear from the fact that a real Schottky group is either Fuchsian group or has a Fuchsian group of index two.

Corollary 2.3. Let $G$ be a real Schottky group of genus $g \geq 2$ with reflection $\tau$. Let $\widehat{G}$ be any group of conformal/anticonformal automorphisms of $\widehat{\mathbb{C}}$ containing $G$ as finite index normal subgroup. Then $[\widehat{G}: G] \leq 24(g-1)$.

Proof. The limit sets of $G$ and $\widehat{G}$ are the same, say $\Lambda$. Let us denote by $\Omega$ their region of discontinuity. We have that $\Lambda \subset C_{\tau}$, where $C_{\tau}$ is the circle of fixed points of $\tau$. It follows that $\tau$ commutes with each element in $\widehat{G}$. On the closed Riemann surface $S=\Omega / G$, of genus $g$, we have the induced reflection $\tau$ and the induced group of automorphisms $\widehat{G} / G<K(S, \tau)$. Since $|K(S, \tau)| \leq 24(g-1)$ by May's results [18] and [19], we are done.

A real Schottky group $G$ is called maximally symmetric if the uniformized surface is maximally symmetric. By the above, this is equivalent to say that there is a finite normal extension $\widehat{G}$ (as group of conformal/anticonformal automorphisms of the Riemann sphere) containing $G$ of index $24(g-1)$. We have that in this case $S / K(S, \tau)=\Omega / \widehat{G}$ must be a closed disc with exactly for branch values, of orders $2,2,2$ and 3 , on its border.

Remark. In the case we have a hyperelliptic Riemann surface $S$ of genus $g \geq 2$, with hyperelliptic involution $j: S \rightarrow S$, which also admits a reflection $\tau: S \rightarrow S$, then the uniqueness of $j$ asserts that $j \in K(S, \tau)$. Take any real Schottky group $G$ uniformizing the surface $S$. We have that $j$ can be 
lifted for such an uniformization. The facts that $j$ has fixed points and has order two assert that we can find a lifting $\widetilde{j}$ of $j$ of order two. Since $S / j$ is the Riemann sphere with $2(g+1)$ branch values of orders 2 , we have that $G$ is also a hyperelliptic Schottky group. We will prove later that there are no maximally symmetric Riemann surfaces of genus $g \geq 3$ which are hyperelliptic.

\section{Riemann matrices and real Schottky groups}

\subsection{Riemann matrices}

An object which also describe closed Riemann surfaces are the Riemann period matrices. These matrices are computed as follows (see for instance [6]). Let $S$ be a closed Riemann surface of genus $g \geq 1$. A basis for homology of $S$, say $\alpha_{1}, \ldots, \alpha_{g}, \beta_{1}, \ldots, \beta_{g}$, is called symplectic if its intersection matrix is

$$
M=\left[\begin{array}{rr}
0 & I \\
-I & 0
\end{array}\right]
$$

where $I$ denotes the $g \times g$ identity matrix. For each symplectic homology basis $\alpha_{1}, \ldots, \alpha_{g}, \beta_{1}, \ldots, \beta_{g}$, there is a unique basis $w_{1}, \ldots, w_{g}$, for the space of holomorphic 1-forms on $S$ so that

$$
\int_{\alpha_{j}} w_{k}= \begin{cases}1, & j=k \\ 0, & j \neq k\end{cases}
$$

The matrix

$$
Z=\left[\begin{array}{ccc}
\int_{\beta_{1}} w_{1} & \cdots & \int_{\beta_{g}} w_{1} \\
\int_{\beta_{1}} w_{2} & \cdots & \int_{\beta_{g}} w_{2} \\
\vdots & \cdots & \vdots \\
\int_{\beta_{1}} w_{g} & \cdots & \int_{\beta_{g}} w_{g}
\end{array}\right]
$$

is a symmetric matrix with positive imaginary part called a Riemann matrix for $S$. In particular, each Riemann matrix is a point in the Siegel space $\mathcal{H}_{g}$, consisting of those symmetric complex matrices of size $g \times g$ with positive imaginary part. The group $\widehat{\operatorname{Sp}_{2 g}(\mathbb{Z})}$, consisting of the integer matrices $T$ of size $2 g \times 2 g$ such that ${ }^{t} T M T= \pm M$, is called the extended symplectic group. Its index two subgroup $\operatorname{Sp}_{2 g}(\mathbb{Z})$, consisting of those $T \in \widehat{\operatorname{Sp}_{2 g}}(\mathbb{Z})$ so that ${ }^{t} T M T=M$, is called the symplectic group. The group $\widehat{\operatorname{Sp}_{2 g}(\mathbb{Z})}$ acts 
on the Siegel space $\mathcal{H}_{g}$ as follows: Let $Z \in \mathcal{H}_{g}$ and

$$
T=\left(\begin{array}{cc}
A & B \\
C & D
\end{array}\right) \in \widetilde{\mathrm{Sp}_{2 g}(\mathbb{Z})}
$$

then

$$
T(Z)=\left\{\begin{array}{lll}
(A+Z C)^{-1}(B+Z D), & \text { if } & T \in \mathrm{Sp}_{2 g}(\mathbb{Z}) \\
(A+\bar{Z} C)^{-1}(B+\bar{Z} D), & \text { if } & T \notin \operatorname{Sp}_{2 g}(\mathbb{Z})
\end{array}\right.
$$

Remark. The above action on the Siegel space is not the classical one which is given as

$$
T(Z)=\left\{\begin{array}{lll}
(A Z+B)(C Z+D)^{-1}, & \text { if } & T \in \mathrm{Sp}_{2 g}(\mathbb{Z}) \\
(A \bar{Z}+B)(C \bar{Z}+D)^{-1}, & \text { if } & T \notin \operatorname{Sp}_{2 g}(\mathbb{Z})
\end{array}\right.
$$

Our action is obtained by writing the homology as

$$
(\alpha \beta)=\left(\alpha_{1} \cdots \alpha_{g} \beta_{1} \cdots \beta_{g}\right)
$$

and the 1 -forms as $w={ }^{t}\left(w_{1} \cdots w_{g}\right)$.

If we start with a different symplectic homology basis of $S$, say $\left\{\widehat{\alpha}_{1}, \ldots, \widehat{\beta}_{g}\right\}$, then we obtain a new Riemann matrix $\widehat{Z}$. Now, there is a symplectic matrix

$$
N=\left(\begin{array}{ll}
A & B \\
C & D
\end{array}\right)
$$

so that

$$
\left[\widehat{\alpha}_{1} \cdots \widehat{\alpha}_{g} \widehat{\beta}_{1} \cdots \widehat{\beta}_{g}\right]=\left[\alpha_{1} \cdots \alpha_{g} \beta_{1} \cdots \beta_{g}\right] N
$$

From this we obtain the equality

$$
\widehat{Z}=(A+Z C)^{-1}(B+Z D)
$$

The reciprocal also holds:

Torelli's Theorem. Two matrices $Z, \widehat{Z} \in \mathcal{H}_{g}$ are Riemann matrices of conformally equivalent Riemann surfaces if and only if there is a symplectic matrix

$$
N=\left(\begin{array}{cc}
A & B \\
C & D
\end{array}\right) \in S p_{2 g}(\mathbb{Z})
$$

so that

$$
\widehat{Z}=(A+Z C)^{-1}(B+Z D) .
$$


Let us assume that $\tau: S \rightarrow S$ is a reflection on $S$, that is, an anticonformal involution of $S$ with fixed points. The induced isomorphism (as $\mathbb{Z}$-module)

$$
\tau: \mathrm{H}_{1}(S, \mathbb{Z}) \rightarrow \mathrm{H}_{1}(S, \mathbb{Z})
$$

has a $\mathbb{Z}$-submodule

$$
\mathrm{H}_{1}(S, \mathbb{Z})_{\tau}=\left\{a \in \mathrm{H}_{1}(S, \mathbb{Z}): \tau(a)=-a\right\}
$$

with dimension equal to $g$. An adapted symplectic homology basis for $S$ is a symplectic homology basis $\left\{\alpha_{1}, \ldots, \alpha_{g}, \beta_{1}, \ldots, \beta_{g}\right\}$, for which $\left\{\alpha_{1}, \ldots, \alpha_{g}\right\}$ is a basis of $\mathrm{H}_{1}(S, \mathbb{Z})_{\tau}$. The Riemann matrix $Z$ obtained from such an adapted basis is called an adapted Riemann matrix of $S$. The (extended) symplectic representation of $\tau$ in such a basis has the form

$$
\rho(\tau)=\left(\begin{array}{cc}
-I & B_{\tau} \\
0 & I
\end{array}\right)
$$

where $B_{\tau} \in \operatorname{Symm}(g, \mathbb{Z})$. If we consider another adapted symplectic homology basis $\left\{\widehat{\alpha}_{1}, \ldots, \widehat{\beta}_{g}\right\}$, with adapted Riemann matrix $\widehat{Z}$, then it is not hard to see that a symplectic matrix $N$ sending one basis on the other must have the form

$$
N=\left(\begin{array}{cc}
A & B \\
0 & { }^{t} A^{-1}
\end{array}\right)
$$

with $A^{-1} B \in \operatorname{Symm}(g, \mathbb{Z})$. From this we have that

$$
\widehat{Z}=A^{-1} B+A^{-1} Z^{t} A^{-1}
$$

This, together Torelli's theorem, gives us the real Torelli's theorem which can be readed as follows [23]:

Real Torelli's Theorem. Two matrices $Z, \widehat{Z} \in \mathcal{H}_{g}$ are adapted Riemann matrices of conformally equivalent pairs $(S, \tau)$ and $(\widehat{S}, \widehat{\tau})$ if and only if there are matrices $A \in G L(g, \mathbb{Z})$ and $Q \in \operatorname{Symm}(g, \mathbb{Z})$ so that

$$
\widehat{Z}=Q+A^{-1} Z^{t} A^{-1}
$$

In general, the explicit computation of Riemann matrices is hard and it is related to the famous Schottky problem: To give a characterization of the Riemann matrices of closed Riemann surfaces inside the space of symmetric matrices with positive imaginary part. In the next section we describe a method of computing "explicitly" Riemann matrices of closed Riemann surfaces admitting a reflection with the help of real Schottky groups and a remark due to Burnside [3]. 


\subsection{Computation of Riemann matrices by real Schottky groups}

This section explains how to use real Schottky groups in order to explicitly compute Riemann matrices of the respective uniformized real surface. Let $G$ be a real Schottky group, with associated reflection $\sigma$, uniformizing a closed Riemann surface $S$, with induced reflection $\tau$. Let us denote by $\Omega$ the region of discontinuity of $G$ and denote by $P: \Omega \rightarrow S$ the natural holomorphic covering with $G$ as covering group. Let us fix some fundamental set of loops for $G$, say $\alpha_{1}, \ldots, \alpha_{g}, \widetilde{\alpha}_{1}, \ldots, \widetilde{\alpha}_{g}$, and let $A_{1}, \ldots, A_{g}$ a set of free generators of $G$ respect to these loops. We give the counterclockwise orientation to the loops $\alpha_{1}, \ldots, \alpha_{g}$, and the induced orientations on $\widetilde{\alpha}_{1}, \ldots, \widetilde{\alpha}_{g}$ by the transformations $A_{1}, \ldots, A_{g}$. Consider any set of pairwise disjoint oriented paths $\beta_{1}, \ldots, \beta_{g}$, so that:

(a) $\beta_{k}$ connects a point $p_{k}^{\text {end }} \in \alpha_{k}$ to the point $p_{k}^{\text {start }}=A_{k}\left(p_{k}^{\text {end }}\right) \in \widetilde{\alpha}_{k}$;

(b) the orientation of $\beta_{k}$ is so that $p_{k}^{\text {start }}$ is the starting point and $p_{k}^{\text {end }}$ is the ending point; and

(c) $\beta_{k}$ is disjoint from $\alpha_{t}$ and $\widetilde{\alpha}_{t}$, for all $t \neq k$.

Let us denote again by $\alpha_{k}$ and $\beta_{k}$ the projections on $S$ of the above respective loops. We obtain in this way a symplectic homology basis for $S$. Let us denote by $w_{1}, w_{2}, \ldots, w_{g}$, the dual basis of holomorphic 1-forms. Let us denote by $Z$ the Riemann matrix for $S$, correspondent to the above symplectic homology basis. The (extended) symplectic representation of $\sigma$ in the above symplectic basis has the form

$$
\rho(\sigma)=\left(\begin{array}{cc}
-I & B_{\sigma} \\
0 & I
\end{array}\right)
$$

Since $\sigma(Z)=Z$, we have that

$$
R e(Z)=\frac{-1}{2} B_{\sigma}
$$

Now, under the assumption that the real Schottky group $G$ has $\infty \in \Omega$, Burnside [3] has remarked that the series

$$
\sum_{\gamma \in G} \gamma^{\prime}(z)
$$

converges uniformly on compact subsets of $\Omega$ to a meromorphic map which is holomorphic on $\Omega-G(\infty)$ and with double poles at each point in $G(\infty)$. A simple argument for this is as follows. In the case that $G$ keeps invariant 
a line $L$ (a circle containing $\infty$ ) is rather simple. In fact, let $V \subset \Omega$ be some compact set and $M=\left\{\gamma_{1}, \ldots, \gamma_{n}\right\} \subset G$ be so that $\gamma_{j}^{-1}(\infty) \in V$. Then there is a positive constant $A>0$ so that

$$
\sum_{\gamma \in G-M}\left|\gamma^{\prime}(z)\right| \leq A \sum_{\gamma \in G} \frac{1}{|c|^{2}}
$$

where $\gamma(z)=\frac{a z+b}{c z+d}, a d-b c=1$. Let us choose a point $q \in L$ and a positive number $R>1$ so that $B=\{z \in \mathbb{C}:|z-q|>R\} \cup\{\infty\}$ satisfies that $\gamma(B) \cap B=\emptyset$ for all $\gamma \in G$ different from the identity. In this case, we have that the sets $\gamma(B)$ are orthogonal discs to $L$ and, in particular,

$$
\sum_{\gamma \in G, \gamma \neq I} \operatorname{diam}(\gamma(B)) \leq 2 R
$$

where diam denotes the Euclidean diameter in the Euclidean plane. We may use the inequality given in C.7 of [15] which in our case reads

$$
\operatorname{diam}(\gamma(B)) \geq \frac{1}{|c|^{2}} \frac{1}{\operatorname{dist}\left(\gamma^{-1}(\infty), B\right)} \geq \frac{1}{|c|^{2}} \frac{1}{R},
$$

for $\gamma \neq I$. Since for $V \subset \Omega-G(\infty)$ we have $M=\emptyset$, the above says that

$$
\sum_{\gamma \in G} \gamma^{\prime}(z)
$$

converges uniformly on $V$ to a holomorphic map. Now, around a point $\gamma_{1}^{-1}(\infty), \gamma \neq I$, we have that the series

$$
\sum_{\gamma \in G-\left\{\gamma_{1}\right\}} \gamma^{\prime}(z)
$$

converges locally to a holomorphic map and, since $\gamma_{1}^{\prime}(z)$ has a double pole in $\gamma_{1}^{-1}(\infty)$, we are done in this case. The case of circles can be carried in the following way. Choose some point $q \in L \cap \Omega$ (then $q \neq \infty$ ). Let

$$
T(z)=\frac{1}{z-q} .
$$

Set $H=T G T^{-1}$ and $L^{\prime}=T(L)$. Then $H$ is a real Schottky group keeping invariant the line $L^{\prime}$ and for which $\infty \in \Omega(H)$. In this case we have the convergence property for $\sum_{h \in H} h^{\prime}(w)$ on compact subsets of $\Omega(H)$. Let $V$ be a compact subset of $\Omega$ so that $q \notin V$. Then for $z \in V$ we have

$$
\sum_{h \in H, h \neq I}\left|h^{\prime}(T(z))\right|=\sum_{\gamma \in G, \gamma \neq I}\left|\gamma^{\prime}(z)\right| \frac{1}{|\gamma(z)-q|^{2}|T(z)|^{2}} .
$$


The fact that $q \notin V$ asserts that

$$
\operatorname{Inf}\left\{\frac{1}{|T(z)|^{2}} ; z \in V\right\}=M>0 .
$$

Also, except for a finite number of elements of $G$, say $\gamma_{1}, \ldots, \gamma_{n}$, we have that

$$
\operatorname{Inf}\left\{\frac{1}{|\gamma(z)-q|^{2}} ; z \in V, \gamma \in G-\left\{\gamma_{1}, \ldots, \gamma_{n}\right\}\right\}=N>0 .
$$

Now it follows that

$$
\sum_{\gamma \in G, \gamma \neq I}\left|\gamma^{\prime}(z)\right| \frac{1}{|\gamma(z)-q|^{2}|T(z)|^{2}} \geq N M \sum_{\gamma \in G, \gamma \neq I}\left|\gamma^{\prime}(z)\right|,
$$

obtaining the desired converge.

The above asserts, in particular, that we can lift each 1 -form $w_{j}$ to $\Omega$ as

$$
\widehat{w}_{j}(z)=\frac{1}{2 \pi i} \sum_{\gamma \in G} \frac{\gamma^{\prime}(z)}{\gamma(z)-A_{j}^{-1}(\infty)} d z,
$$

for $j=1, \ldots, g$. These liftings have the property that

$$
\int_{\alpha_{k}} \widehat{w}_{j}= \begin{cases}0, & j=r \\ 1, & j \neq r\end{cases}
$$

We must observe at this point that the different possible choices for the oriented paths $\beta_{1}, \ldots, \beta_{g}$ are not uniquely determined by the points $p_{j}^{\text {start }}$ and $p_{j}^{\text {end }}$. But the different possible choices have the property that the imaginary part of the integrals $\int_{\beta_{j}} \widehat{w}_{k}$ are in fact uniquely determined by these points. It follows that the imaginary part of the Riemann matrix $Z$ is given uniquely by $\operatorname{Im}(Z)=Y=\left(y_{k j}\right)$, where

$$
\begin{aligned}
y_{k j} & =\frac{1}{2 \pi} \operatorname{Re}\left(\log \left(\prod_{\gamma \in G} \frac{\gamma\left(p_{k}^{\text {start }}\right)-A_{j}^{-1}(\infty)}{\gamma\left(p_{k}^{\text {end }}\right)-A_{j}^{-1}(\infty)}\right)\right) \\
& =\frac{1}{2 \pi} \log \left(\prod_{\gamma \in G} \frac{\left|\gamma\left(p_{k}^{\text {start }}\right)-A_{j}^{-1}(\infty)\right|}{\left|\gamma\left(p_{k}^{\text {end }}\right)-A_{j}^{-1}(\infty)\right|}\right)
\end{aligned}
$$

The above method then gives us an explicit way for computation of Riemann matrices of real Riemann surfaces. In fact, the above can be done with a computer program. How fast is the above algorithm and how it can be improved will be discussed elsewhere. 


\section{Maximally symmetric Riemann surfaces}

Let us consider a closed Riemann surface $S$ admitting a reflection $\tau: S \rightarrow S$. We may think of $S$ as the quotient surface of a real Schottky group $G$ for which $\tau$ is the induced reflection asociated to $G$. Let us assume the order of $K(S, \tau)$ is exactly $24(g-1)$, that is, $S$ is maximally symmetric Riemann surface (we also say that $\tau$ is a maximal reflection) [8]. In this case, we have that $S / K(S, \tau)$ is a closed disc with exactly four branch values, of orders 2, 2, 2 and 3, on the border. A list of the maximally symmetric Riemann surface of genus at most 40 is given in [20]. Part of the list is the following (all families depends on one real parameter):

(1) In genus two there are two families of maximally symmetric Riemann surfaces. In the first family the reflection $\tau$ has three components of fixed points. In the second family the reflection $\tau$ has exactly one component of fixed points, which divides $S$ into two one-holes tori. In both cases, the quotient $S / \tau$ are orientable. In the first case is a threeholed sphere and in the second a one-holed torus. In subsection 4.1. we describe them in a more explicit way.

(2) In genus three there are two families. In the first one $\tau$ has four components of fixed points (the quotient $S / \tau$ is a 4 -holed sphere). In the second family $\tau$ has exactly three components of fixed points and $S / \tau$ is a three-holed projective plane. These are described in subsection 4.2 .

(3) In genus four there is exactly one family, The reflection $\tau$ has three components of fixed points and $S / \tau$ is a three-holed torus.

(4) In genus five there are two families. In the first family the reflection $\tau$ has four components of fixed points and $S / \tau$ is four-holed torus. In the second family, $\tau$ has six components of fixed points and $S / \tau$ is six-holed sphere.

An important class of Riemann surfaces are the hyperelliptic ones. The following asserts that there are no maximally symmetric Riemann surface inside the hyperelliptic locus, for genus at least 3. A different proof of this fact can be find in [2].

Proposition 4.1. A maximally symmetric Riemann surface of genus greater or equal to three cannot be hyperelliptic.

Proof. Let $S$ be a maximally symmetric Riemann surface $S$ of genus $g \geq 3$ together a maximal reflection $\tau: S \rightarrow S$. The quotient $S / K(S, \tau)$ is a closed disc with four branched values of orders 2, 2, 2 and 3 on its border. Let us also assume $S$ to be hyperelliptic with $j: S \rightarrow S$ the hyperelliptic involution. 
The uniqueness of $j$ implies that $j \in K^{+}(S, \tau)$, the index two subgroup of conformal automorphisms of $K(S, \tau)$. Let us consider the two-fold-branched covering $Q: S \rightarrow \widehat{\mathbb{C}}$ induced by the hyperelliptic involution. The maximal reflection $\tau: S \rightarrow S$ descends to a reflection $\eta: \widehat{\mathbb{C}} \rightarrow \widehat{\mathbb{C}}$ commuting with each automorphism in $N=K^{+}(S, \tau) / j$, a group of Möbius transformations of order $6(g-1)$. Let us denote by $\mathcal{C}$ the circle of fixed points of $\eta$. Then the group $N$ preserves $\mathcal{C}$ and, in particular, it is either a Fuchsian group or has an index two subgroup that is Fuchsian. In either case, we have inside $N$ a Fuchsian group $N_{0}$ of order $3(g-1) \geq 6$. But a finite Fuchsian group is necessarily a cyclic group and, in particular, $N_{0}$ is a cyclic group of order $3(g-1)$. It follows that $N_{0}$ must have a fixed point of order $3(g-1)$. But $S / K(S, \tau)$ only has branch values of orders 2 and 3 . It follows that $3(g-1) \leq 3$, a contradiction.

\subsection{Genus two maximally symmetric Riemann surfaces}

The proof of proposition 4.1., also permits us to determine the maximally symmetric Riemann surfaces of genus two. In fact, as observed in the above proof, since $S / K(S, \tau)$ has no branched values of order 6 and $N$ has order 6 , $N$ cannot be Fuchsian. We have then that $N$ has an index two subgroup $N_{0}$ which is Fuchsian. $N_{0}$ is a cyclic group of order 3 which permutes the 6 branched values of order 2 of $Q$. We have two possibilities: (i) all branched values of order 2 are contained in $\mathcal{C}$ or (ii) none is contained in there (in which case, three of them are contained in the interior of one of the discs bounded by $\mathcal{C}$ and the other three are their images by $\eta$ ). In case (i) we have that $\tau: S \rightarrow S$ is a reflection with exactly 3 components of fixed points, dividing $S$ into two sub-surfaces, each one a three-holed sphere. The group $K^{+}(S, \tau)$ is generated by the symmetric group in 3 letters $\mathcal{S}_{3}$ (the stabilizer in $K^{+}(S, \tau)$ of one of these sub-surfaces) and the hyperelliptic involution $j$. The group $K(S, \tau)$ is then the group generated by $\tau$ and $K^{+}(S, \tau)$. In case (ii) we have that $\tau$ is a reflection on $S$ with exactly one circle of fixed points, dividing $S$ into two sub-surfaces, each one a genus one surface with a boundary. In this case, $K^{+}(S, \tau)$ is generated by a a cyclic group of order 6 (the stabilizer in $K^{+}(S, \tau)$ of one of these sub-surfaces), and a conformal involution permuting both sub-surfaces (the fixed points are contained in the fixed points of $\tau$ ). The group $K(S, \tau)$ is then the group generated by $\tau$ and $K^{+}(S, \tau)$. The algebraic curves corresponding to these surfaces are given by:

$$
y^{2}=\left(x^{3}-\lambda^{3}\right)\left(x^{3}-1 / \lambda^{3}\right)
$$

where either $\lambda>1$, if we are in the case (ii) or $\lambda=e^{i \eta}, \eta \in(0, \pi / 3)$, if we are in case (i). 
In this case, $K(S, \tau)=\langle\tau, j, \alpha, \beta\rangle$, where

$$
\begin{gathered}
\tau=\left(\begin{array}{rrr}
x & \mapsto & 1 / \bar{x} \\
y & \mapsto & \bar{y} / \bar{x}^{3}
\end{array}\right) ; j=\left(\begin{array}{rrr}
x & \mapsto & x \\
y & \mapsto & -y
\end{array}\right) ; \\
\alpha=\left(\begin{array}{rrrr}
x & \mapsto & e^{2 \pi i / 3} x \\
y & \mapsto & y
\end{array}\right) ; \beta=\left(\begin{array}{rrr}
x & \mapsto & 1 / x \\
y & \mapsto & y / x^{3}
\end{array}\right)
\end{gathered}
$$

\section{Remarks.}

(1) Let us note that in the case $\eta=\pi / 6$ the curve is given by

$$
y^{2}=x^{6}+1,
$$

isomorphic to $w^{2}=z^{6}-1$ (by the change of variables $w=i y$ and $\left.z=e^{i \pi / 6} x\right)$, has the extra automorphism

$$
\alpha^{1 / 2}=\left(\begin{array}{rrr}
x & \mapsto & e^{\pi i / 3} x \\
y & \mapsto & y
\end{array}\right)
$$

and, in particular, the extra automorphisms

$$
\tau_{3}=\left(\begin{array}{lll}
x & \mapsto & \bar{x} \\
y & \mapsto & \bar{y}
\end{array}\right) ; j^{1 / 2}=\left(\begin{array}{llr}
x & \mapsto & 1 / x \\
y & \mapsto & i y / x^{3}
\end{array}\right)
$$

(2) Let us observe that each maximally symmetric Riemann surface $(S, \tau)$ of genus 2, for which $S / \tau$ is a three-holed sphere, has a reflection $\tau^{\prime} \in K(S, \tau)$ so that $S / \tau^{\prime}$ is a one-holed torus. Such a reflection corresponds to the conjugation $x \mapsto \bar{x}$ in the above corresponding algebraic curve. By direct inspection, one can see that $K\left(S, \tau^{\prime}\right)$ is not maximal.

(3) Since the Weierstrass points of one of the families belong to the unit circle and the ones for the other are not contained in any circle or line, we see that the projection into the moduli space of genus 2 of these two families define disjoint sets.

\subsection{Genus three maximally symmetric Riemann surfaces}

If we consider a maximally symmetric Riemann surface $S$ of genus 3 , then $K(S, \tau)$ has order 48 and $K^{+}(S, \tau)$ has order 24 . Since $S$ cannot be hyperelliptic, the only possibility for $K^{+}(S, \tau)$ is to be isomorphic to the symmetric group on four letters $\mathcal{S}_{4}$. In this case, these surfaces are described by the following algebraic curves [21]:

$$
x^{4}+y^{4}+z^{4}+\lambda\left(x^{2} y^{2}+y^{2} z^{2}+z^{2} x^{2}\right)=0,
$$

where $\lambda \in(-\infty,-2)$ for case $(\mathrm{v})$ and $\lambda \in(-2,-1)$ for case (ii). 
The reflection $\tau$ is given by $\tau[x: y: z]=[\bar{x}, \bar{y}, \bar{z}]$, and $K^{+}(S, \tau)$ is the symmetric group in four letters $\mathcal{S}_{4}$ generated by the projective transformations

$$
a=\left(\begin{array}{rrr}
0 & -1 & 0 \\
1 & 0 & 0 \\
0 & 0 & 1
\end{array}\right) ; b=\left(\begin{array}{lll}
1 & 0 & 0 \\
0 & 0 & 1 \\
0 & 1 & 0
\end{array}\right)
$$

\section{Real Schottky uniformizations of maximal symmetric Riemann surfaces}

\subsection{Uniformization of the closed disc with branch values of orders $2,2,2,3$ on its border}

Let us consider a maximally symmetric Riemann surface $S$ with a maximal reflection $\tau: S \rightarrow S$. As observed before, $S / K(S, \tau)$ is a closed disc with four branched values of orders $2,2,2$ and 3 on its border. For each $p \in(2-\sqrt{3}, 1)$, let us consider the group $\widehat{K}_{p}$ generated by the following reflections (see fig. 1 ):

$$
\sigma_{1}(z)=\bar{z}, \quad \sigma_{2}(z)=e^{\frac{2 \pi i}{3}} \bar{z}, \quad \sigma_{p}(z)=\frac{\left(1+p^{2}\right) \bar{z}-2 p}{2 p \bar{z}-\left(1+p^{2}\right)} \text { and } \sigma(z)=\frac{1}{\bar{z}} .
$$

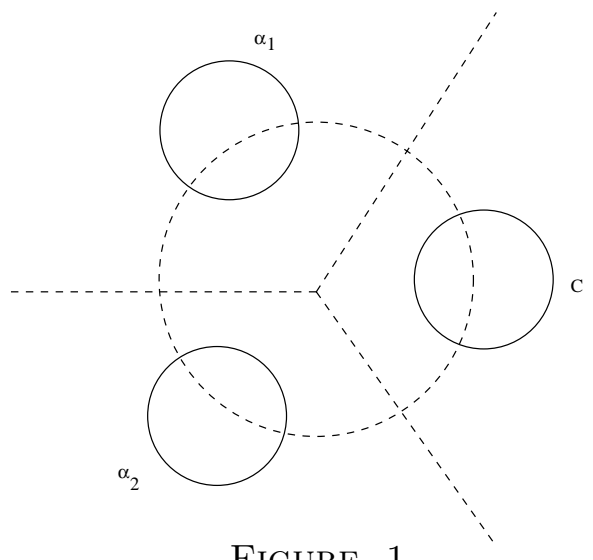

If we set $W(z)=\sigma_{2} \sigma_{1}(z)=e^{\frac{2 \pi i}{3}} z, T(z)=\sigma \sigma_{1}(z)=\frac{1}{z}$ and

$$
J(z)=\sigma_{p} \sigma_{1}(z)=\frac{\left(p+\frac{1}{p}\right) z-2}{2 z-\left(p+\frac{1}{p}\right)},
$$

then the group $\widehat{K}_{p}$ is also generated by the transformations $T, W, J$ and $\sigma$. As consequence of Klein-Maskit's combination theorems [15], we have

$$
\begin{gathered}
\widehat{K}_{p}=\left\langle T, W, J, \sigma: T^{2}=W^{3}=(W T)^{2}=J^{2}=(T J)^{2}=\sigma^{2}=(\sigma T)^{2}=\right. \\
\left.\sigma W \sigma W^{-1}=(\sigma J)^{2}=1\right\rangle .
\end{gathered}
$$


The Kleinian group $\widehat{K}_{p}$ has a connected region of discontinuity $\Omega$ and a fundamental domain for it is determined the the bounded region determined by the unit circle, the two rays of arguments $\pi i / 3$ and $-\pi i / 3$, respectively, and the circle $C$ orthogonal to the unit circle and containing the points $p$ and $1 / p$ (see figure 1 ). The Klein surface uniformized by $\widehat{K}$ is the closed unit disc with exactly four branch values of orders $2,2,2$ and 3 on its border.

Theorem 5.1. Every Klein surface which is the unit closed disc with exactly four branch values of orders 2, 2, 2 and 3 on the border can be uniformized by a group $\widehat{K}_{p}$ for some $p \in(2-\sqrt{3}, 1)$.

Proof. This is just consequence of quasiconformal deformation theory and the fact that every reflection on the Riemann sphere has an Euclidean circle as fixed points.

The index two subgroup $K_{p}$ of $\widehat{K}_{p}$ of the orientation preserving transformations is generated by the transformations $T, W$ and $J$ and the presentation of $K_{p}$ is

$$
K_{p}=\left\langle T, W, J: T^{2}=W^{3}=(W T)^{2}=J^{2}=(T J)^{2}=1\right\rangle,
$$

that is, isomorphic to the free amalgamated product of the dihedral group $D_{3}=\langle T, W\rangle$ with the Klein group $\mathbb{Z}_{2}+\mathbb{Z}_{2}=\langle T, J\rangle$ over the cyclic group of order two generated by $T$. The Riemann surface $\Omega / K_{p}$ is the Riemann sphere with four branched values of order 2, 2, 2 and 3 , all of them fixed by the reflection induced by $\sigma$ on it. A fundamental domain of $K_{p}$ can be considered as the union of the above fundamental domain of $\widehat{K}_{p}$ with its image under $\sigma$ together the points of the unit circle located between $e^{\frac{\pi i}{3}}$ and $e^{\frac{-\pi i}{3}}$, respectively.

\section{Remarks.}

(1) The boundary case $p=2-\sqrt{3}$ corresponds to the case that $\widehat{K}_{p}$ uniformizes a hyperbolic closed triangle with one vertex at infinity, one vertex with angle $\pi / 3$ and the other with angle $\pi / 2$. The corresponding group $K_{p}$ uniformizes an sphere with three branched values of orders 2,3 and $\infty$, respectively.

(2) The boundary case $p=1$ corresponds to the case that $\widehat{K}_{p}$ is only generated by the reflections $\sigma_{1}, \sigma_{2}$ and $\sigma$, but acting on the threepunctured Riemann sphere. In this case, the group $\widehat{K}_{p}$ uniformizes the closed unit disc with three branched values at the border with orders 2,3 and $\infty$, respectively. 


\subsection{Maximally symmetric real Schottky groups}

For each $p \in(2-\sqrt{3}, 1)$, we consider the Kleinian group $\widehat{K}=\widehat{K}_{p}$. We proceed to construct certain maximally symmetric real Schottky groups of genus $g \in\{2,3,4,5\}$. Then we show that every maximally symmetric Riemann surface of genus $g \in\{2,3,4,5\}$ can be uniformized by one of these.

\subsubsection{Schottky uniformizations of genus 2}

Consider the Möbius transformations $A_{1}=J W J W^{-1}, A_{2}=J W^{-1} J W$, $B_{1}=\left(\sigma_{p} \sigma_{2}\right)^{2}$ and $B_{2}=\sigma_{1} B_{1} \sigma_{1}$. The groups $G_{2}=\left\langle A_{1}, A_{2}\right\rangle$ and $F_{2}=$ $\left\langle B_{1}, B_{2}\right\rangle$ are real Schottky groups of genus two and a standard fundamental domain for both $G_{2}$ and $F_{2}$ is given by the circles $W(C), J(W(C)), W^{-1}(C)$ and $J\left(W^{-1}(C)\right)$. Since $W A_{1} W^{-1}=A_{1}^{-1} A_{2}, W A_{2} W^{-1}=A_{1}^{-1}, J A_{1} J=$ $A_{1}^{-1}, J A_{2} J=A_{2}^{-1}, T A_{1} T^{-1}=A_{2}, T A_{2} T=A_{1}, \sigma A_{1} \sigma=A_{1}, \sigma A_{2} \sigma=$ $A_{2}, W B_{1} W^{-1}=B_{1}^{-1} B_{2}, W B_{2} W^{-1}=B_{1}^{-1}, J B_{1} J=B_{2}^{-1}, J B_{2} J=B_{1}^{-1}$, $T B_{1} T=B_{2}, T B_{2} T=B_{1}, \sigma B_{1} \sigma=B_{1}$ and $\sigma B_{2} \sigma=B_{2}$, we have that both $G_{2}$ and $F_{2}$ are normal subgroups of $\widehat{K}$.

Moreover, for $Q \in\left\{G_{2}, F_{2}\right\}, \widehat{K} / Q \cong D_{3}+\mathbb{Z}_{2}+\mathbb{Z}_{2}$ and $K / Q \cong D_{3}+\mathbb{Z}_{2}$. In particular, the Riemann surfaces of genus two $S_{2}=\Omega / G_{2}$ (respectively, $R_{2}=$ $\Omega / F_{2}$ ) admit the group $\widehat{K} / G_{2}$ (respectively, $\widehat{K} / F_{2}$ ) as group of symmetries, with $K / G_{2}$ (respectively, $K / F_{2}$ ) as its index two subgroup of orientation preserving symmetries. The quotient $S_{2} /\left(\widehat{K} / G_{2}\right)$ (respectively, $R_{2} /\left(\widehat{K} / F_{2}\right)$ ) is the unit closed disc with four branch values in its boundary of orders 2 , 2,2 and 3 .

\subsubsection{Schottky uniformizations of genus 3}

Let us consider the group $G_{3}$ generated by the transformations $D_{1}=$ $\left(\sigma_{p} \sigma_{2}\right)^{3}, D_{2}=W D_{1} W^{-1}$ and $D_{3}=W^{-1} D_{1} W$, and the group $F_{3}$ generated by the transformations $E_{1}=\sigma \sigma_{p} \sigma_{2} \sigma_{1} \sigma_{2} \sigma_{p} \sigma_{2} \sigma_{1} \sigma_{p} \sigma_{2}, E_{2}=W B_{1} W^{-1}$ and $E_{3}=W^{-1} B_{1} W$. It is easy to see that $G_{3}$ and $F_{3}$ are Schottky groups of genus three with standard fundamental domain bounded by the circles $C_{1}=\sigma_{p}\left(\sigma_{2}(C)\right), C_{6}=\sigma_{1}\left(C_{1}\right), C_{2}=W\left(C_{6}\right), C_{3}=W\left(C_{1}\right), C_{5}=W^{-1}\left(C_{1}\right)$ and $C_{4}=W^{-1}\left(C_{6}\right)$, where $C=\operatorname{Fix}\left(\sigma_{p}\right)$.

The relations $\sigma_{1} D_{1} \sigma_{1}=D_{3}^{-1}, \sigma_{1} D_{2} \sigma_{1}=D_{2}^{-1}, \sigma_{2} D_{1} \sigma_{2}=D_{1}^{-1}, \sigma_{2} D_{2} \sigma_{2}=$ $D_{3}^{-1}, \sigma_{p} D_{1} \sigma_{p}=D_{1}^{-1}, \sigma_{p} D_{2} \sigma_{p}=D_{1} D 2 D_{3}, \sigma_{p} D_{3} \sigma_{p}=D_{3}^{-1}, \sigma D_{1} \sigma=D_{1}$, $\sigma D_{2} \sigma=D_{2}, \sigma D_{3} \sigma=D_{3}, \sigma_{1} E_{1} \sigma_{1}=E_{3}^{-1}, \sigma_{1} E_{2} \sigma_{1}=E_{2}^{-1}, \sigma_{2} E_{1} \sigma_{2}=E_{1}^{-1}$, $\sigma_{2} E_{2} \sigma_{2}=E_{3}^{-1}, \sigma_{p} E_{1} \sigma_{p}=E_{3}, \sigma_{p} E_{2} \sigma_{p}=E_{3}^{-1} E 2^{-1} E_{1}^{-1}, \sigma E_{1} \sigma=E_{1}, \sigma E_{2} \sigma=$ $E_{2}, \sigma E_{3} \sigma=E_{3}$, show the normality of these two groups.

A direct computation implies that $\widehat{K} / G_{3}$ and $\widehat{K} / F_{3}$ are groups of order 48 and non-isomorphic. 


\subsubsection{Schottky uniformizations of genus 4}

Let $G_{4}$ be the subgroup of $G_{2}$ generated by the transformations $U_{1}=A_{1}^{3}$, $U_{2}=A_{1} A_{2}, U_{3}=A_{2} A_{1}^{-2}$ and $U_{4}=A_{1}^{2} A_{2} A_{1}^{-1}$. This group is exactly the smallest normal subgroup of $G_{2}$ having the elements $U_{1}$ and $U_{2}$ and has index 3. The group $G_{4}$ is a free group of index three in the real Schottky group $G_{2}$ and then it is a real Schottky group of genus 4 . Since $G_{2}$ is subgroup of index 24 in $\widehat{K}$ and $G_{4}$ has index 3 in $G_{2}$, we have that $G_{4}$ is a subgroup of index 72 of $\widehat{K}$. The relations $W U_{1} W^{-1}=U_{1}^{-1} U_{4} U_{3} U_{2}, W U_{2} W^{-1}=U_{1}^{-1} U_{4}$, $W U_{3} W^{-1}=U_{1}^{-1} U_{3}^{-1} U_{4}^{-1} U_{1}, W U_{4} W^{-1}=U_{1}^{-1} U_{4} U_{3} U_{4}^{-1} U_{1}, J U_{1} J^{-1}=U_{1}^{-1}$, $J U_{2} J^{-1}=U_{1}^{-1} U_{3}^{-1}, J U_{3} J^{-1}=U_{2}^{-1} U_{1}, J U_{4} J^{-1}=U_{1}^{-1} U_{4}^{-1} U_{1}, T U_{1} T^{-1}=$ $U_{3} U_{4} U_{2}, T U_{2} T^{-1}=U_{3} U_{1}, T U_{3} T^{-1}=U_{4}^{-1} U_{3}^{-1}, T U_{4} T^{-1}=U_{3} U_{4} U_{3}^{-1}$ and the fact that $\sigma$ commutes with every transformation in $G_{4}$, assert that $G_{4}$ is a normal subgroup of $\widehat{K}$.

\subsubsection{Schottky uniformizations of genus 5}

Let $G_{5}$ (respectively, $F_{5}$ ) be the subgroup of $G_{2}$ (respectively, $F_{2}$ ) generated by the square of all the elements in $G_{2}$ (respectively, $F_{2}$ ), that is:

$$
G_{5}=\left\langle x^{2}: x \in G_{2}\right\rangle, \quad F_{5}=\left\langle x^{2}: x \in F_{2}\right\rangle .
$$

Since $G_{2}$ (respectively, $F_{2}$ ) is normal subgroup of index 24 in $\widehat{K}$ and $G_{5}$ (respectively, $F_{5}$ ) is normal of index 4 in $G_{2}$ (respectively, $F_{2}$ ), we have that $G_{5}$ (respectively, $F_{5}$ ) is a normal subgroup of $\widehat{K}$ of index 96 . The group $G_{5}$ (respectively, $F_{5}$ ) is a free group of index four in the real Schottky group $G_{2}$ (respectively, $F_{2}$ ) and then it is a real Schottky group of genus 5 . Free generators for $G_{5}$ (respectively, $F_{5}$ ) are given by $T_{1}=A_{1}^{2}, T_{2}=A_{2}^{2}, T_{3}=$ $A_{1}^{-1} A_{2}^{2} A_{1}, T_{4}=A_{1}^{-1} A_{2}^{-1} A_{1} A_{2}$ and $T_{5}=A_{2}^{-1} A_{1} A_{2} A_{1}$ (respectively, $R_{1}=B_{1}^{2}$, $R_{2}=B_{2}^{2}, R_{3}=B_{1}^{-1} B_{2}^{2} B_{1}, R_{4}=B_{1}^{-1} B_{2}^{-1} B_{1} B_{2}$ and $R_{5}=B_{2}^{-1} B_{1} B_{2} B_{1}$ ).

Theorem 5.2. For each maximally symmetric Riemann surface $S$ with a maximal reflection $\tau: S \rightarrow S$ there is a suitable value of $p \in(2-\sqrt{3}, 1)$ so that $S$ is uniformized by

(a) $G_{2}$ or $F_{2}$, if $g=2$;

(b) $G_{3}$, if $S / \tau$ is orientable and $g=3$;

(c) $F_{3}$, if $S / \tau$ is non-orientable and $g=3$;

(d) $G_{4}$, if $g=4$; and

(e) $G_{5}$ or $F_{5}$, if $S / \tau$ is orientable and $g=5$.

Proof. We proceed to prove only (a) since the other situations are simmilar. Fix a value of $p \in(2-\sqrt{3}, 1)$ and let us consider the Kleinian group $K=K_{p}$. Let $S$ be a maximally symmetric Riemann surface of genus two together a maximal reflection $\tau: S \rightarrow S$. The topological action of $K(S, \tau)$ is either reflected by $G_{2}$ or $F_{2}$, since there are exactly two possible actions. 
Without lost of generality, we may assume that this is reflected by $G_{2}$. Set $S_{2}=\Omega / G_{2}$ and let $f: S_{2} \rightarrow S$ be an orientation preserving homeomorphisms so that $f \widehat{K} / G_{2} f^{-1}=K(S, \tau)$. We may assume that $f$ is quasiconformal homeomorphisms with complex Beltrami differential $\mu$. We lift $\mu$ to $\Omega$ and extend it as zero to the limit set of $\widehat{K}$. Let $W: \widehat{\mathbb{C}} \rightarrow \widehat{\mathbb{C}}$ be a $\mu$-quasiconformal homeomorphisms. Then $W \widehat{K} W^{-1}$ is again a group generated by reflections. We may assume that $W$ fixes 0,1 and $\infty$. Since a reflection has as fixed points only Euclidean circles on the Riemann sphere, we have that the group $W \widehat{K} W^{-1}$ is again one of our groups $\widehat{K}$ for a suitable value of $p$. If we denote by $\pi: \Omega \rightarrow \Omega / G_{2}$ the holomorphic covering induced by $G_{2}$, then we have that $f \pi W^{-1}: \Omega\left(W \widehat{K} W^{-1}\right) \rightarrow S$ an uniformization of $S$ by the corresponding Schottky group $G_{2}$, so that the lifting of $K(S, \tau)$ is exactly $W \widehat{K} W^{-1}$.

\section{Remarks.}

(1) In the case $p=2-\sqrt{3}$, the group $G_{2}$ is a noded Schottky group of genus two uniformizing an stable Riemann surface of genus two with exactly one dividing node. In the case $p=1$, we can think of the group $G_{2}$ as the trivial group acting on the three-punctured sphere, that is, uniformizing the three-punctured sphere.

(2) In the case $p=2-\sqrt{3}$, the group $F_{2}$ is a noded Schottky group of genus two uniformizing an stable Riemann surface of genus two with exactly three non-dividing nodes. In the case $p=1$, we can think of the group $F_{2}$ as the trivial group acting on the three-punctured sphere, that is, uniformizing the three-punctured sphere.

(3) In the case $p=2-\sqrt{3}$, the group $G_{3}$ is a noded Schottky group of genus three uniformizing a stable Riemann surface of genus three with exactly four non-dividing nodes and two components, each one a four punctured sphere. In the case $p=1$, we can think of the group $G_{3}$ as the trivial group acting on the three-punctured sphere, that is, uniformizing the three-punctured sphere. For $p \in(2-\sqrt{3}, 1)$, the respective groups $G_{3}$ uniformize the algebraic curves corresponding to $\lambda \in(-2,-1)$; for $p=2-\sqrt{3}$ it uniformizes the algebraic curve corresponding to $\lambda=-1$; and for $p=1$ it uniformizes the algebraic curve for $\lambda=-2$.

(4) In the case $p=2-\sqrt{3}$, the group $F_{3}$ is a noded Schottky group of genus three uniformizing a stable Riemann surface of genus three with exactly three non-dividing nodes and one component, an sphere with six punctures. In the case $p=1$, we can think of the group $F_{3}$ as the trivial group acting on the three-punctured sphere, that is, uniformizing the three-punctured sphere. For $p \in(2-\sqrt{3}, 1)$, the respective groups 
$F_{3}$ uniformize the algebraic curves corresponding to $\lambda \in(-\infty,-2)$; for $p=2-\sqrt{3}$ it uniformizes the algebraic curve corresponding to $\lambda=-\infty$; and for $p=1$ it uniformizes the algebraic curve for $\lambda=-2$.

(5) In the case $p=2-\sqrt{3}$, the group $G_{4}$ is a noded Schottky group uniformizing a stable Riemann surface of genus four with exactly three non-dividing nodes and two components, each one of genus one.

(6) In the case $p=2-\sqrt{3}$, the group $G_{5}$ is a noded Schottky group uniformizing a stable Riemann surface of genus five with exactly four non-dividing nodes and two components, each one of genus one. Similarly, the group $F_{5}$ is a noded Schottky group uniformizing a stable Riemann surface of genus five with exactly six non-dividing nodes.

\section{Riemann matrices and algebraic curve of maximal symmetric Riemann surfaces}

In this section we proceed to compute adapted Riemann matrices of maximal symmetric Riemann surfaces of genus 2,3,4 and 5, with the help of the real Schottky uniformizations of last section and Burnside's remark. We also construct algebraic curves for such kind of surfaces in genus 2, 3 and 4 .

\subsection{Genus 2 maximal symmetric Riemann surfaces}

We start with the following classical result in genus two.

Theorem 6.1. Let $S$ be a maximal symmetric Riemann surface of genus 2 with a maximal reflection $\tau: S \rightarrow S$. Let $p \in(2-\sqrt{3}, 1)$ be so that

(i) $S=\Omega / G_{2}$, if $S / \tau$ is a genus one surface with one hole;

(ii) $S=\Omega / F_{2}$, if $S / \tau$ is a genus zero surface with three holes.

$A$ Riemann matrix of $S$ is given by $Z=P+i Q$, with

$$
P=\left\{\begin{array}{ll}
0 & \text { in case (ii), } \\
\frac{1}{2}\left[\begin{array}{ll}
0 & 1 \\
1 & 0
\end{array}\right] \quad \text { in case (i), }
\end{array} \quad Q=\frac{w}{2}\left[\begin{array}{ll}
2 & 1 \\
1 & 2
\end{array}\right],\right.
$$

where

$$
\begin{gathered}
w=\left\{\begin{array}{l}
\frac{1}{2 \pi} \log \left(\prod_{\gamma \in G_{2}} \frac{\left|\gamma\left(q_{2}\right)-A_{1}^{-1}(\infty)\right|}{\left|\gamma\left(q_{1}\right)-A_{1}^{-1}(\infty)\right|}\right) \quad \text { in case (i), } \\
\frac{1}{2 \pi} \log \left(\prod_{\gamma \in F_{2}} \frac{\left|\gamma\left(p_{2}\right)-B_{1}^{-1}(\infty)\right|}{\left|\gamma\left(q_{1}\right)-B_{1}^{-1}(\infty)\right|}\right) \quad \text { in case (ii), }
\end{array}\right. \\
q_{1}=e^{\left(\frac{2 \pi}{3}-\theta\right) i}, q_{2}=J\left(q_{1}\right), p_{2}=\sigma_{p}\left(q_{1}\right) \text { and } \theta \in\left(0, \frac{\pi}{2}\right) \text { is such that } \cos \theta=\frac{2 p}{1+p^{2}} .
\end{gathered}
$$


Proof. Case (i): Set $\alpha_{1}=W(C), \alpha_{1}^{\prime}=J\left(\alpha_{1}\right), \alpha_{2}=W^{-1}(C)$ and $\alpha_{2}^{\prime}=$ $J\left(\alpha_{2}\right)$. If we orient the circles $\alpha_{1}, \alpha_{2}, \alpha_{1}^{\prime}$ and $\alpha_{2}^{\prime}$ and consider the oriented paths $\beta_{1}$ and $\beta_{2}$ as shown in figure 2 , then the projection of these loops and paths determines an adapted symplectic homology basis on the closed Riemann surface $S_{2}$.
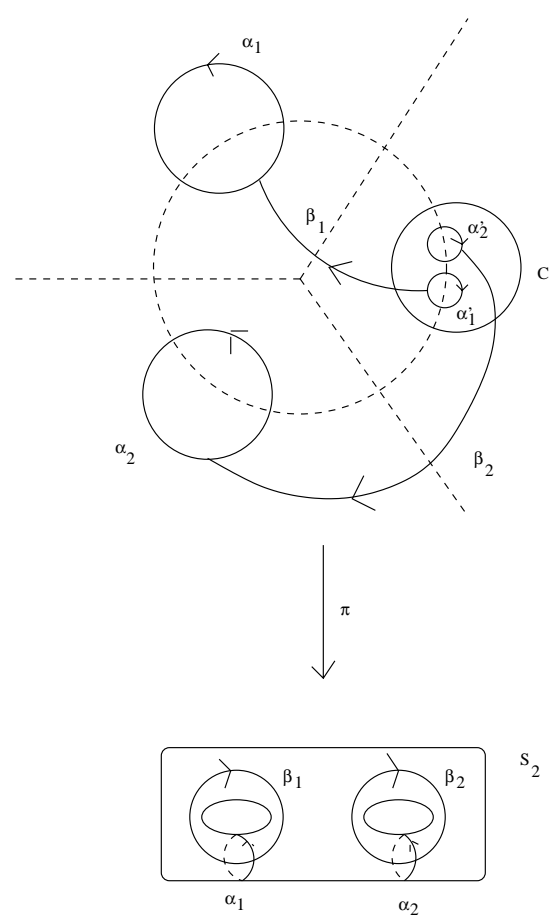

FIGURE 2

In this way, we obtain a faithful symplectic representation $\rho: \widehat{K} / G_{2} \rightarrow$ $\widehat{\operatorname{Sp}(4 ; \mathbb{Z})}$ defined by

$$
\begin{gathered}
\rho(\sigma)=\left[\begin{array}{rrrr}
-1 & 0 & 0 & -1 \\
0 & -1 & -1 & 0 \\
0 & 0 & 1 & 0 \\
0 & 0 & 0 & 1
\end{array}\right], \quad \rho(T)=\left[\begin{array}{llll}
0 & 1 & 0 & 0 \\
1 & 0 & 0 & 0 \\
0 & 0 & 0 & 1 \\
0 & 0 & 1 & 0
\end{array}\right] \\
\rho(J)=\left[\begin{array}{rrrr}
-1 & 0 & 0 & 0 \\
0 & -1 & 0 & 0 \\
0 & 0 & -1 & 0 \\
0 & 0 & 0 & -1
\end{array}\right], \quad \rho(W)=\left[\begin{array}{rrrr}
0 & -1 & a & b \\
1 & -1 & c & d \\
0 & 0 & -1 & -1 \\
0 & 0 & 1 & 0
\end{array}\right]
\end{gathered}
$$

The Riemann matrix $Z=X+i Y \in \mathcal{H}_{2}$ of $S_{2}$ defined by the above symplectic basis is a fixed point of the symplectic group $\rho\left(\widehat{K} / G_{2}\right)$. It follows that: $a=-1, b=0, c=0, d=1$, and

$$
Z=\frac{1}{2}\left[\begin{array}{ll}
0 & 1 \\
1 & 0
\end{array}\right]+i\left[\begin{array}{cc}
w & \frac{w}{2} \\
\frac{w}{2} & w
\end{array}\right], \quad \text { where } w>0 .
$$


The results due to Burnside [3] described at the end of section 3 can be used in this case to the group $G_{2}$ to compute the value of $w$ in function of $p$ to obtain the desired result in this case.

Case (ii): We proceed in analogous way as done for the previous case. Set $\alpha_{1}=W(C), \alpha_{1}^{\prime}=\sigma_{p}\left(\alpha_{1}\right), \alpha_{2}=W^{-1}(C)$ and $\alpha_{2}^{\prime}=\sigma_{p}\left(\alpha_{2}\right)$. If we orient the circles $\alpha_{1}, \alpha_{2}, \alpha_{1}^{\prime}$ and $\alpha_{2}^{\prime}$ and consider the oriented paths $\beta_{1}$ and $\beta_{2}$ as shown in figure 3 , then the projection of these loops and paths determines an adapted symplectic homology basis on the closed Riemann surface $R_{2}$.

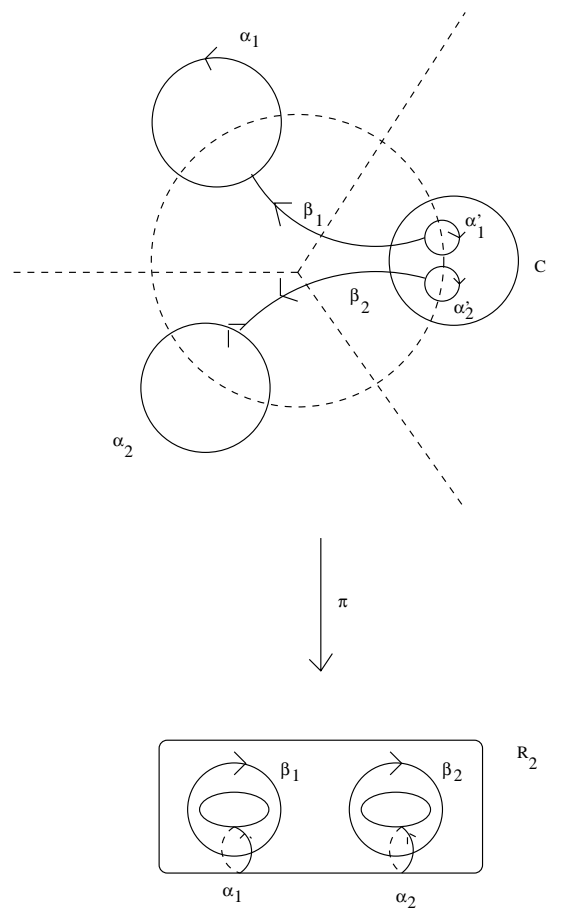

FIGURE 3

In this way, we obtain a faithful symplectic representation

$$
\rho: \widehat{K} / F_{2} \rightarrow \widehat{\operatorname{Sp}(4 ; \mathbb{Z})}
$$

defined by

$$
\begin{gathered}
\rho(\sigma)=\left[\begin{array}{rrrr}
-1 & 0 & 0 & 0 \\
0 & -1 & 0 & 0 \\
0 & 0 & 1 & 0 \\
0 & 0 & 0 & 1
\end{array}\right], \quad \rho(T)=\left[\begin{array}{llll}
0 & 1 & 0 & 0 \\
1 & 0 & 0 & 0 \\
0 & 0 & 0 & 1 \\
0 & 0 & 1 & 0
\end{array}\right] \\
\rho(J)=\left[\begin{array}{rrrr}
0 & -1 & 0 & 0 \\
-1 & 0 & 0 & 0 \\
0 & 0 & 0 & -1 \\
0 & 0 & -1 & 0
\end{array}\right], \quad \rho(W)=\left[\begin{array}{rrrr}
0 & -1 & a & b \\
1 & -1 & c & d \\
0 & 0 & -1 & -1 \\
0 & 0 & 1 & 0
\end{array}\right]
\end{gathered}
$$


The Riemann matrix $Z=X+i Y \in \mathcal{H}_{2}$ of $R_{2}$ defined by the above symplectic basis is a fixed point of the symplectic group $\rho\left(\widehat{K} / F_{2}\right)$. It follows that: $a=b=c=d=0$, and

$$
Z=i\left[\begin{array}{cc}
w & \frac{w}{2} \\
\frac{w}{2} & w
\end{array}\right]
$$

where $w>0$. Again the results due to Burnside [3] described at the end of section 3 can be used in this case to the group $F_{2}$ to compute the value of $w$ in function of $p$ to obtain the result.

\subsection{Algebraic curves of maximally symmetric surfaces of genus 2}

Let us note at this point that the results in [6] permit us to describe the algebraic curve (using theta functions) of the surfaces $S_{2}$ and $R_{2}$ in function of $w$ and, in particular, of $p$. This can be done without the use of Theta functions as follows. For each $p \in(2-\sqrt{3}, 1)$ we have explicitly $w_{1}$ and $w_{2}$ in the region $\Omega$ for $G_{2}$ and for $F_{2}$ (see at the end of section 3.2.). We have then the explicit holomorphic map $F: \Omega \rightarrow \widehat{\mathbb{C}}$, defined by

$$
F(z)=\frac{w_{1}(z)}{w_{2}(z)}
$$

which is a branched covering for the group generated by the elliptic transformations

(a) $J, W J W^{-1}$ and $W^{-1} J W$, for the group $F_{2}$;

(b) $T, W T W^{-1}$ and $W^{-1} T W$, for the group $G_{2}$.

This is a lift of the two-fold-cover (induced by the hyperelliptic involution) over the Riemann sphere. We have that the algebraic curve for the surface in question is given by

$$
y^{2}=(x-a)(x-b)(x-c)(x-d)(x-e)(x-f)
$$

where

$$
\begin{array}{r}
a=F(k), \quad b=F(W(k)), \quad c=F\left(W^{2}(k)\right), \\
d=F\left(\frac{1}{k}\right), \quad e=F\left(W\left(\frac{1}{k}\right)\right), \quad f=F\left(W^{2}\left(\frac{1}{k}\right)\right)
\end{array}
$$

where

$$
k= \begin{cases}p & \text { for } G_{2} \\ e^{i \theta} & \text { for } F_{2}\end{cases}
$$




\subsection{Example}

In the particular case $\lambda=e^{\pi i / 6}$ in section 4.1 , which corresponds to the algebraic curve

$$
y^{2}=x^{6}+1,
$$

we may use the Riemann period matrix as computed in theorem 6.1., in order to obtain the value of $p$ corresponding to it. In fact, using the symplectic homology basis as considered above for $F_{2}$, we can describe the symplectic representation of the automorphism $j^{1 / 2}$, which is in this case:

$$
\rho\left(j^{1 / 2}\right)=\left[\begin{array}{rrrr}
0 & 0 & -1 & -1 \\
0 & 0 & -1 & 0 \\
0 & 1 & 0 & 0 \\
1 & -1 & 0 & 0
\end{array}\right]
$$

In this way, since the matrix $Z$ (the one of theorem 6.1. (ii)) is fixed by $\rho\left(j^{1 / 2}\right)$, we obtain the equality

$$
\frac{4 \pi}{\sqrt{3}}=\log \left(\prod_{\gamma \in F_{2}} \frac{\left|\gamma\left(p_{2}\right)-B_{1}^{-1}(\infty)\right|}{\left|\gamma\left(B_{1}^{-1}\left(q_{1}\right)\right)-B_{1}^{-1}(\infty)\right|}\right)
$$

Using words of lenght at most 8 in the free group, we obtain the approximation value $p \cong 0.75659027099609375$. This has been done thanks to a program in Mathematica written by J. Figueroa. In fact, with such a program we are able to numerically compute the respective adapted Riemann matrix in function of $p$ in genus 2 . The program can be adapted easily for greater genus. We have not tested the stability of such a program.

\subsection{Genus 3 maximal symmetric Riemann surfaces}

For the group $G_{3}$ we choose $\alpha_{1}=C_{2}$ counterclockwise oriented, $\alpha_{1}^{\prime}=C_{1}$ with the orientation of $\alpha_{1}$ induced by $D_{1}$. We set $\alpha_{2}=W\left(\alpha_{1}\right), \alpha_{3}=W^{-1}\left(\alpha_{1}\right)$, $\alpha_{2}^{\prime}=W\left(\alpha_{1}^{\prime}\right), \alpha_{3}^{\prime}=W^{-1}\left(\alpha_{1}^{\prime}\right)$. We also consider pairwise disjoint oriented simple paths $\beta_{1}, \beta_{2}$ and $\beta_{3}$ as shown in figure 4 .

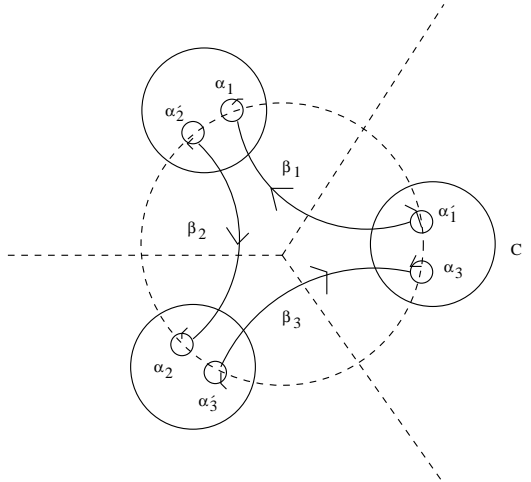

FIGURE 4 
For the group $F_{3}$ we choose $\alpha_{1}=C_{3}$ counterclockwise oriented, $\alpha_{1}^{\prime}=C_{6}$ with the orientation of $\alpha_{1}$ induced by $E_{1}$. We set $\alpha_{2}=W\left(\alpha_{1}\right), \alpha_{3}=$ $W^{-1}\left(\alpha_{1}\right), \alpha_{2}^{\prime}=W\left(\alpha_{1}^{\prime}\right), \alpha_{3}^{\prime}=W^{-1}\left(\alpha_{1}^{\prime}\right)$. We also consider pairwise disjoint oriented simple paths $\beta_{1}, \beta_{2}$ and $\beta_{3}$ as shown in figure 5 .

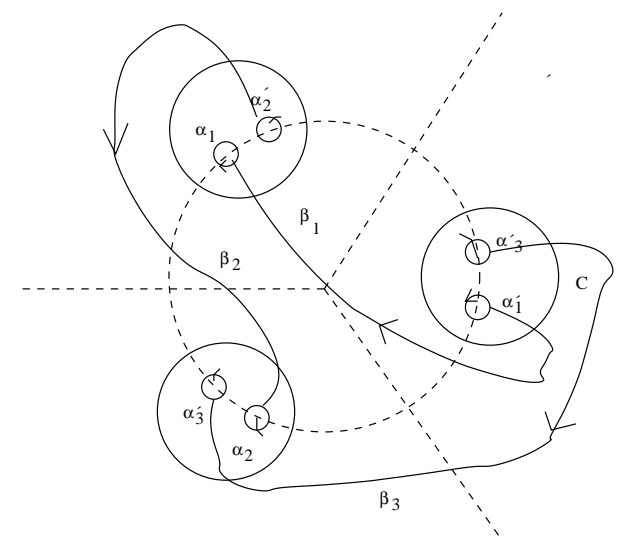

FIGURE 5

On each of the above two cases, the oriented loops $\alpha_{1}, \alpha_{2}, \alpha_{3}$, and the oriented paths $\beta_{1}, \beta_{2}$ and $\beta_{3}$ induce an adapted symplectic homology basis on the uniformized Riemann surface $S$. Symplectic representations

$$
\theta_{1}: \widehat{K} / G_{3} \rightarrow \widetilde{\operatorname{Sp}_{6}(\mathbb{Z})}, \quad \theta_{2}: \widehat{K} / F_{3} \rightarrow \widetilde{\operatorname{Sp}_{6}(\mathbb{Z})}
$$

are then easy to read as follows:

$$
\begin{aligned}
& \theta_{1}(\sigma)=\left[\begin{array}{rrrrrr}
-1 & 0 & 0 & 0 & 0 & 0 \\
0 & -1 & 0 & 0 & 0 & 0 \\
0 & 0 & -1 & 0 & 0 & 0 \\
0 & 0 & 0 & 1 & 0 & 0 \\
0 & 0 & 0 & 0 & 1 & 0 \\
0 & 0 & 0 & 0 & 0 & 1
\end{array}\right], \theta_{1}(T)=\left[\begin{array}{rrrrrr}
0 & 0 & -1 & 0 & 0 & 0 \\
0 & -1 & 0 & 0 & 0 & 0 \\
-1 & 0 & 0 & 0 & 0 & 0 \\
0 & 0 & 0 & 0 & 0 & -1 \\
0 & 0 & 0 & 0 & -1 & 0 \\
0 & 0 & 0 & -1 & 0 & 0
\end{array}\right] \\
& \theta_{1}(J)=\left[\begin{array}{rrrrrr}
0 & 0 & 1 & 0 & 0 & 0 \\
-1 & -1 & -1 & 0 & 0 & 0 \\
1 & 0 & 0 & 0 & 0 & 0 \\
0 & 0 & 0 & 0 & -1 & 1 \\
0 & 0 & 0 & 0 & -1 & 0 \\
0 & 0 & 0 & 1 & -1 & 0
\end{array}\right], \theta_{1}(W)=\left[\begin{array}{llllll}
0 & 0 & 1 & 0 & 0 & 0 \\
1 & 0 & 0 & 0 & 0 & 0 \\
0 & 1 & 0 & 0 & 0 & 0 \\
0 & 0 & 0 & 0 & 0 & 1 \\
0 & 0 & 0 & 1 & 0 & 0 \\
0 & 0 & 0 & 0 & 1 & 0
\end{array}\right] \\
& \theta_{2}(\sigma)=\left[\begin{array}{rrrrrr}
-1 & 0 & 0 & -1 & -1 & 1 \\
0 & -1 & 0 & -1 & 1 & -1 \\
0 & 0 & -1 & 1 & -1 & -1 \\
0 & 0 & 0 & 1 & 0 & 0 \\
0 & 0 & 0 & 0 & 1 & 0 \\
0 & 0 & 0 & 0 & 0 & 1
\end{array}\right], \theta_{2}(T)=\left[\begin{array}{rrrrrr}
0 & 0 & -1 & 0 & 0 & 0 \\
0 & -1 & 0 & 0 & 0 & 0 \\
-1 & 0 & 0 & 0 & 0 & 0 \\
0 & 0 & 0 & 0 & 0 & -1 \\
0 & 0 & 0 & 0 & -1 & 0 \\
0 & 0 & 0 & -1 & 0 & 0
\end{array}\right]
\end{aligned}
$$




$$
\theta_{2}(J)=\left[\begin{array}{rrrrrr}
-1 & 0 & 0 & 0 & -1 & 0 \\
1 & 1 & 1 & 1 & 0 & 1 \\
0 & 0 & -1 & 0 & -1 & 0 \\
0 & 0 & 0 & -1 & 1 & 0 \\
0 & 0 & 0 & 0 & 1 & 0 \\
0 & 0 & 0 & 0 & 1 & -1
\end{array}\right], \theta_{2}(W)=\left[\begin{array}{rrrrrr}
0 & 0 & 1 & -1 & 1 & 2 \\
1 & 0 & 0 & 1 & 2 & -1 \\
0 & 1 & 0 & 2 & -1 & 1 \\
0 & 0 & 0 & 0 & 0 & 1 \\
0 & 0 & 0 & 1 & 0 & 0 \\
0 & 0 & 0 & 0 & 1 & 0
\end{array}\right]
$$

Now following the same arguments and computations as done for the case of genus two maximal symmetric Riemann surfaces one obtains:

Theorem 6.2. Let $S$ be a maximal symmetric Riemann surface of genus 3 with a maximal reflection $\tau: S \rightarrow S$. Let $p \in(2-\sqrt{3}, 1)$ be so that

(i) $S=\Omega / G_{3}$, if $S / \tau$ is orientable

(ii) $S=\Omega / F_{3}$, if $/ \tau$ is non-orientable.

$A$ Riemann matrix of $S$ is given by $Z=P+i Q$, where

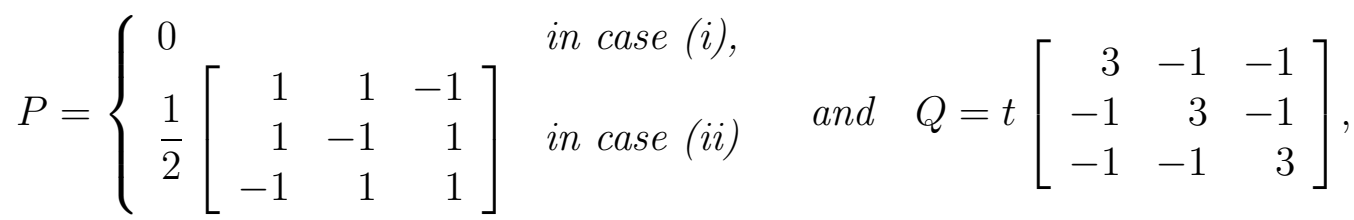

where

$$
t=t_{p}= \begin{cases}\frac{1}{6 \pi} \log \left(\prod_{\gamma \in G_{3}} \frac{\left|\gamma\left(r_{p}\right)-A_{1}^{-1}(\infty)\right|}{\left|\gamma\left(s_{p}\right)-A_{1}^{-1}(\infty)\right|}\right) & \text { in case (i) } \\ \frac{1}{6 \pi} \log \left(\prod_{\gamma \in G_{3}} \frac{\left|\gamma\left(\sigma_{1}\left(r_{p}\right)\right)-B_{1}^{-1}(\infty)\right|}{\left|\gamma\left(l_{p}\right)-B_{1}^{-1}(\infty)\right|}\right) & \text { in case (ii) }\end{cases}
$$

$r_{p}=\sigma_{p}\left(e^{\left(\frac{2 \pi}{3}-\theta\right) i}\right), \quad s_{p}=\sigma_{2}\left(r_{p}\right), \quad l_{p}=\sigma_{2}\left(\sigma_{1}\left(\sigma_{p}\left(\sigma_{2}\left(e^{(-\theta) i}\right)\right)\right)\right)$, and $\theta \in\left(0, \frac{\pi}{2}\right)$ is such that $\cos \theta=\frac{2 p}{1+p^{2}}$.

\subsection{Algebraic curves for maximally symmetric surfaces of genus 3}

Given a value of $p \in(2-\sqrt{3}, 1)$, we have explictly defined on $\Omega$ a basis of holomorphic one forms $w_{1}, w_{2}, w_{3}$ (see section 3.7.) for either $G_{3}$ or $F_{3}$. Since the surfaces uniformized by these groups are non-hyperelliptic ones (see proposition 4.1.), the explicit holomorphic map $F: \Omega \rightarrow \mathbb{C} P^{2}$ defined as

$$
F(z)=\left[w_{1}(z): w_{2}(z): w_{3}(z)\right]
$$

permits to obtain a non-singular projective quartic $\mathcal{Q}$ representing the uniformized surface. To be more precise, we proceed to work the case of $G_{3}$ 
(the case $F_{3}$ can be done in a similar fashion). In this case, we have the projective realizations

$$
\begin{aligned}
\sigma[u: v: w] & =[\bar{u}: \bar{v}: \bar{w}] \\
\sigma_{1}[u: v: w] & =[\bar{w}: \bar{v}: \bar{u}] \\
\sigma_{2}[u: v: w] & =[\bar{u}: \bar{w}: \bar{v}] \\
\sigma_{p}[u: v: w] & =[\bar{u}-\bar{v}: \bar{w}: \bar{w}-\bar{v}]
\end{aligned}
$$

where $[u: v: w]$ are the coordinates of $\mathbb{C} P^{2}$. The form of $\sigma$ asserts that the coefficients of the $\mathcal{Q}$ are all reals. Using the other realizations we obtain that

$$
\begin{aligned}
\mathcal{Q}= & A u^{4}-\frac{4}{3} A u^{3} v+B u^{2} v^{2}-\frac{4}{3} A u v^{3}+A v^{4}-\frac{4}{3} A u^{3} w+ \\
& +(2 A-B) u^{2} v w+(2 A-B) u v^{2} w-\frac{4}{3} A v^{3} w+B u^{2} w^{2}+ \\
& +(2 A-B) u v w^{2}+B v^{2} w^{2}-\frac{4}{3} A u w^{3}-\frac{4}{3} A v w^{3}+A w^{4}
\end{aligned}
$$

To obtain $A$ and $B$, we proceed to evaluate $F$ at some point on $\Omega$; for instance let us consider $z=e^{\pi i / 3}$. Let us assume that $F\left(e^{\pi i / 3}\right)=[a: b: c]$, where $a, b, c \in \mathbb{R}$. Using words of lenght less than $L$, for some choice of a positive integer $L$, we may get numerical approximations of these values. When we plug them into $\mathcal{Q}$ we obtain that

$$
B=A P(a, b, c) / Q(a, b, c)
$$

where $P(a, b, c)=\left(3 a^{4}-4 a^{3}(b+c)+6 a^{2} b c+a\left(-4 b^{3}+6 b^{2} c+6 b c^{2}\right)-4 c^{3}+\right.$ $\left.3 b^{4}-4 b^{3} c-4 b c^{3}+3 c^{4}\right)$ and $Q(a, b, c)=3\left(a^{2}\left(b^{2}+c^{2}-b c\right)-a b c(b+c)+b^{2} c^{2}\right)$. We may assume $A=1$ to get $\mathcal{Q}$.

\subsection{Genus 4 maximal symmetric Riemann surfaces}

Let us consider $\alpha_{1}, \alpha_{2}, \alpha_{1}^{\prime}$ and $\alpha_{2}^{\prime}$ as done for $G_{2}$. A standard fundamental domain for $G_{4}$ is the region bounded by the circles $\gamma_{1}=\alpha_{1}, \gamma_{2}=\alpha_{2}, \gamma_{3}=$ $A_{1}^{2}\left(\alpha_{2}\right), \gamma_{4}=A_{1}\left(\alpha_{2}\right), \gamma_{1}^{\prime}=A_{1}^{3}\left(\alpha_{1}\right), \gamma_{2}^{\prime}=A_{1}\left(\alpha_{2}^{\prime}\right), \gamma_{3}^{\prime}=\alpha_{2}^{\prime}$ and $\gamma_{4}^{\prime}=A_{1}^{2}\left(\alpha_{2}^{\prime}\right)$. We give the orientations induced from the ones given to the loops $\alpha_{1}$ and $\alpha_{2}$. We can easily check that, by choose of a suitable set of pairwise disjoint oriented simple arcs, we get the extended symplectic representation

$$
\theta: \widehat{K} / G_{4} \rightarrow \widehat{\operatorname{Sp}_{8}(\mathbb{Z})}
$$

given by

$$
\begin{gathered}
\theta(\sigma)=\left(\begin{array}{rr}
-I & S \\
0 & I
\end{array}\right) ; \quad \theta(W)=\left(\begin{array}{rr}
W_{1} & Q_{W} \\
0 & { }^{t} W_{1}^{-1}
\end{array}\right) ; \\
\theta(J)=\left(\begin{array}{rr}
J_{1} & Q_{J} \\
0 & { }^{t} J_{1}
\end{array}\right) ; \quad \theta(T)=\left(\begin{array}{rr}
T_{1} & Q_{T} \\
0 & { }^{t} T_{1}
\end{array}\right),
\end{gathered}
$$


where

$$
\begin{gathered}
S=\left(\begin{array}{rrrr}
0 & -1 & 1 & -1 \\
-1 & 0 & -1 & 0 \\
-1 & 1 & 0 & 1 \\
-1 & 0 & -1 & 0
\end{array}\right) ; \quad W_{1}=\left(\begin{array}{rrrr}
0 & -1 & -1 & -1 \\
1 & -1 & 0 & -1 \\
0 & 0 & 0 & 1 \\
0 & 0 & -1 & -1
\end{array}\right) ; \\
J_{1}=\left(\begin{array}{rrrr}
-1 & 0 & 0 & 0 \\
-1 & 0 & -1 & 0 \\
1 & -1 & 0 & 0 \\
0 & 0 & 0 & -1
\end{array}\right) ; \quad T_{1}\left(\begin{array}{rrrr}
0 & 1 & 1 & 1 \\
1 & 0 & 1 & 0 \\
0 & 0 & -1 & -1 \\
0 & 0 & 0 & 1
\end{array}\right),
\end{gathered}
$$

and

$$
Q_{W}=\frac{1}{2}\left(S^{t} W_{1}^{-1}-W_{1} S\right), \quad Q_{J}=\frac{1}{2}\left(S^{t} J_{1}-J_{1} S\right), \quad Q_{T}=\frac{1}{2}\left(S^{t} T_{1}-T_{1} S\right) .
$$

Direct computations permit us to obtain:

Theorem 6.3. Let $S$ be a maximal symmetric Riemann surface of genus 4 with a maximal reflection $\tau: S \rightarrow S$. Let $p \in(2-\sqrt{3}, 1)$ be so that $S=\Omega / G_{4}$. Then a Riemann matrix of $S$ is given by

$$
Z=\frac{-1}{2}\left[\begin{array}{rrrr}
0 & -1 & 1 & -1 \\
-1 & 0 & -1 & 0 \\
-1 & 1 & 0 & 1 \\
-1 & 0 & -1 & 0
\end{array}\right]+i\left[\begin{array}{rrrr}
2(v-u) & v-u & u-v & v-u \\
v-u & -2 u & -u & v \\
u-v & u & -2 u & u \\
v-u & v & u & -2 u
\end{array}\right]
$$

The values of $u$ and $v$ can be written explicitly in function of $p$ as is done in the other cases.

\subsection{Genus 5 maximal symmetric Riemann surfaces}

A standard fundamental domain for $G_{5}$ (respectively, $F_{5}$ ) respect to the above free generators is determined by the circles $\theta_{1}=A_{1}^{-1}\left(\alpha_{1}\right), \theta_{1}^{\prime}=\alpha_{1}^{\prime}$, $\theta_{2}=A_{2}^{-1}\left(\alpha_{2}\right), \theta_{2}^{\prime}=\alpha_{2}^{\prime}, \theta_{3}=A_{1}^{-1} A_{2}^{-1}\left(\alpha_{2}\right), \theta_{3}^{\prime}=A_{1}^{-1}\left(\alpha_{2}^{\prime}\right), \theta_{4}=A_{2}^{-1}\left(\alpha_{1}\right)$, $\theta_{4}^{\prime}=A_{1}^{-1} A_{2}^{-1}\left(\alpha_{1}^{\prime}\right), \theta_{5}=A_{1}^{-1} A_{2}^{-1}\left(\alpha_{1}\right)$ and $\theta_{5}^{\prime}=A_{2}^{-1}\left(\alpha_{1}^{\prime}\right)$ (respectively, $\theta_{1}=$ $B_{1}^{-1}\left(\alpha_{1}\right), \theta_{1}^{\prime}=\alpha_{1}^{\prime}, \theta_{2}=B_{2}^{-1}\left(\alpha_{2}\right), \theta_{2}^{\prime}=\alpha_{2}^{\prime}, \theta_{3}=B_{1}^{-1} B_{2}^{-1}\left(\alpha_{2}\right), \theta_{3}^{\prime}=B_{1}^{-1}\left(\alpha_{2}^{\prime}\right)$, $\theta_{4}=B_{2}^{-1}\left(\alpha_{1}\right), \theta_{4}^{\prime}=B_{1}^{-1} B_{2}^{-1}\left(\alpha_{1}^{\prime}\right), \theta_{5}=B_{1}^{-1} B_{2}^{-1}\left(\alpha_{1}\right)$ and $\left.\theta_{5}^{\prime}=B_{2}^{-1}\left(\alpha_{1}^{\prime}\right)\right)$. We can give to these $\theta$-loops the orientations determined by the orientations we have given to $\alpha_{1}, \alpha_{2}, \alpha_{1}^{\prime}$ and $\alpha_{2}^{\prime}$. Let us choose the following paths: $\delta_{1}=$ $\beta_{1} \cup A_{1}^{-1}\left(\beta_{1}\right), \delta_{2}=\beta_{2} \cup A_{2}^{-1}\left(\beta_{2}\right), \delta_{3}=A_{1}^{-1}\left(\delta_{2}\right)$ (respectively, $\delta_{1}=\beta_{1} \cup B_{1}^{-1}\left(\beta_{1}\right)$, $\left.\delta_{2}=\beta_{2} \cup B_{2}^{-1}\left(\beta_{2}\right), \delta_{3}=B_{1}^{-1}\left(\delta_{2}\right)\right)$, with the orientations determined by the orientations given to $\beta_{1}$ and $\beta_{2}$. For $j=4,5$, we choose oriented paths $\delta_{j}$ contained inside the unit disc starting at $\theta_{j}$ and ending at $\theta_{j}^{\prime}$ and disjoint 
from $\delta_{1}$ and $\delta_{9-j}$. The projections of the above loops and paths determine on the Riemann surface $S_{5}=\Omega\left(G_{5}\right) / G_{5}$ (respectively, $R_{5}=\Omega\left(F_{5}\right) / F_{5}$ ) an adapted symplectic homology basis. Since we have the following relations:

(a) $J T_{1} J=T_{1}^{-1}, J T_{2} J=T_{2}^{-1}, J T_{3} J=T_{1} T_{3}^{-1} T_{1}^{-1}, J T_{4} J=T_{1} T_{3} T_{5}^{-1} T_{2}^{-1}$, $J T_{5} J=T_{2} T_{4}^{-1} T_{3}^{-1} T_{1}^{-1}, J R_{1} J=R_{2}^{-1}, J R_{2} J=R_{1}^{-1}, J R_{3} J=R_{2} R_{4}^{-1}$ $R_{5}^{-1} R_{2}^{-1}, J R_{4} J=R_{2} R_{5} R_{3}^{-1} R_{1}^{-1}, J R_{5} J=R_{1} R_{5}^{-1} R_{2}^{-1} ;$

(b) $W T_{1} W^{-1}=T_{3} T_{5}^{-1}, W T_{2} W^{-1}=T_{1}^{-1}, W T_{3} W^{-1}=T_{4}^{-1} T_{5}^{-1}, W T_{4} W^{-1}=$ $T_{5} T_{1}^{-1}, W T_{5} W^{-1}=T_{2} T_{4}^{-1} T_{5}^{-1}, W R_{1} W^{-1}=R_{3} R_{5}^{-1}, W R_{2} W^{-1}=R_{1}^{-1}$, $W R_{3} W^{-1}=R_{4}^{-1} R_{5}^{-1}, W R_{4} W^{-1}=R_{5} R_{1}^{-1}, W R_{5} W^{-1}=R_{2} R_{4}^{-1} R_{5}^{-1}$;

(c) $T T_{1} T=T_{2}, T T_{2} T=T_{1}, T T_{3} T=T_{5} T_{4}, T T_{4} T=T_{4}^{-1}, T T_{5} T=T_{3} T_{4}$, $T R_{1} T=R_{2}, T R_{2} T=R_{1}, T R_{3} T=R_{5} R_{4}, T R_{4} T=R_{4}^{-1}, T R_{5} T=$ $R_{3} R_{4}$

(d) $\sigma T_{j} \sigma=T_{j}, \sigma R_{j} \sigma=R_{j}, j=1, \ldots, 5$,

we obtain faithful symplectic representations

$$
\rho: \widehat{K} / G_{5} \rightarrow \widehat{\operatorname{Sp}(10 ; \mathbb{Z})} \quad \text { and } \quad \eta: \widehat{K} / F_{5} \rightarrow \widehat{\operatorname{Sp}(10 ; \mathbb{Z})}
$$

defined by

$$
\begin{aligned}
\rho(\sigma) & =\left[\begin{array}{rrrrrrrrrr}
-1 & 0 & 0 & 0 & 0 & 0 & -1 & -1 & 0 & 0 \\
0 & -1 & 0 & 0 & 0 & -1 & 0 & 0 & 0 & -1 \\
0 & 0 & -1 & 0 & 0 & -1 & 0 & 0 & 0 & -1 \\
0 & 0 & 0 & -1 & 0 & 0 & 0 & 0 & 0 & 0 \\
0 & 0 & 0 & 0 & -1 & 0 & -1 & -1 & 0 & 0 \\
0 & 0 & 0 & 0 & 0 & 1 & 0 & 0 & 0 & 0 \\
0 & 0 & 0 & 0 & 0 & 0 & 1 & 0 & 0 & 0 \\
0 & 0 & 0 & 0 & 0 & 0 & 0 & 1 & 0 & 0 \\
0 & 0 & 0 & 0 & 0 & 0 & 0 & 0 & 1 & 0 \\
0 & 0 & 0 & 0 & 0 & 0 & 0 & 0 & 0 & 1
\end{array}\right] \\
\eta(\sigma) & =\left[\begin{array}{rrrrrrrrrr}
-1 & 0 & 0 & 0 & 0 & 0 & 0 & 0 & 0 & 0 \\
0 & -1 & 0 & 0 & 0 & 0 & 0 & 0 & 0 & 0 \\
0 & 0 & -1 & 0 & 0 & 0 & 0 & 0 & 0 & 0 \\
0 & 0 & 0 & -1 & 0 & 0 & 0 & 0 & 0 & 0 \\
0 & 0 & 0 & 0 & -1 & 0 & 0 & 0 & 0 & 0 \\
0 & 0 & 0 & 0 & 0 & 1 & 0 & 0 & 0 & 0 \\
0 & 0 & 0 & 0 & 0 & 0 & 1 & 0 & 0 & 0 \\
0 & 0 & 0 & 0 & 0 & 0 & 0 & 1 & 0 & 0 \\
0 & 0 & 0 & 0 & 0 & 0 & 0 & 0 & 1 & 0 \\
0 & 0 & 0 & 0 & 0 & 0 & 0 & 0 & 0 & 1
\end{array}\right]
\end{aligned}
$$




$$
\begin{aligned}
& \rho(T)=\eta(T)=\left[\begin{array}{rrrrrrrrrr}
0 & 1 & 0 & 0 & 0 & t_{11} & t_{12} & t_{13} & t_{14} & t_{15} \\
1 & 0 & 0 & 0 & 0 & t_{21} & t_{22} & t_{23} & t_{24} & t_{25} \\
0 & 0 & 0 & 1 & 1 & t_{31} & t_{32} & t_{33} & t_{34} & t_{35} \\
0 & 0 & 0 & -1 & 0 & t_{41} & t_{42} & t_{43} & t_{44} & t_{45} \\
0 & 0 & 1 & 1 & 0 & t_{51} & t_{52} & t_{53} & t_{54} & t_{55} \\
0 & 0 & 0 & 0 & 0 & 0 & 1 & 0 & 0 & 0 \\
0 & 0 & 0 & 0 & 0 & 1 & 0 & 0 & 0 & 0 \\
0 & 0 & 0 & 0 & 0 & 0 & 0 & 0 & 0 & 1 \\
0 & 0 & 0 & 0 & 0 & 0 & 0 & 1 & -1 & 1 \\
0 & 0 & 0 & 0 & 0 & 0 & 0 & 1 & 0 & 0
\end{array}\right] \\
& \rho(J)=\left[\begin{array}{rrrrrrrrrr}
-1 & 0 & 0 & 0 & 0 & j_{11} & j_{12} & j_{13} & j_{14} & j_{15} \\
0 & -1 & 0 & 0 & 0 & j_{21} & j_{22} & j_{23} & j_{24} & j_{25} \\
0 & 0 & -1 & 0 & 0 & j_{31} & j_{32} & j_{33} & j_{34} & j_{35} \\
1 & -1 & 1 & 0 & -1 & j_{41} & j_{42} & j_{43} & j_{44} & j_{45} \\
-1 & 1 & -1 & -1 & 0 & j_{51} & j_{52} & j_{53} & j_{54} & j_{55} \\
0 & 0 & 0 & 0 & 0 & -1 & 0 & 0 & 1 & -1 \\
0 & 0 & 0 & 0 & 0 & 0 & -1 & 0 & -1 & 1 \\
0 & 0 & 0 & 0 & 0 & 0 & 0 & -1 & 1 & -1 \\
0 & 0 & 0 & 0 & 0 & 0 & 0 & 0 & 0 & -1 \\
0 & 0 & 0 & 0 & 0 & 0 & 0 & 0 & -1 & 0
\end{array}\right] \\
& \eta(J)=\left[\begin{array}{rrrrrrrrrr}
0 & -1 & 0 & 0 & 0 & j_{11} & j_{12} & j_{13} & j_{14} & j_{15} \\
-1 & 0 & 0 & 0 & 0 & j_{21} & j_{22} & j_{23} & j_{24} & j_{25} \\
0 & 0 & 0 & -1 & -1 & j_{31} & j_{32} & j_{33} & j_{34} & j_{35} \\
-1 & 1 & -1 & 0 & 1 & j_{41} & j_{42} & j_{43} & j_{44} & j_{45} \\
1 & -1 & 0 & 0 & -1 & j_{51} & j_{52} & j_{53} & j_{54} & j_{55} \\
0 & 0 & 0 & 0 & 0 & 0 & -1 & 0 & -1 & 1 \\
0 & 0 & 0 & 0 & 0 & -1 & 0 & 0 & 1 & -1 \\
0 & 0 & 0 & 0 & 0 & 0 & 0 & 0 & -1 & 0 \\
0 & 0 & 0 & 0 & 0 & 0 & 0 & -1 & 0 & 0 \\
0 & 0 & 0 & 0 & 0 & 0 & 0 & -1 & 1 & -1
\end{array}\right] \\
& \rho(W)=\eta(W)=\left[\begin{array}{rrrrrrrrrr}
0 & -1 & 0 & 0 & 0 & w_{11} & w_{12} & w_{13} & w_{14} & w_{15} \\
0 & 0 & -1 & 0 & 1 & w_{21} & w_{22} & w_{23} & w_{24} & w_{25} \\
1 & -1 & 0 & 1 & 0 & w_{31} & w_{32} & w_{33} & w_{34} & w_{35} \\
0 & 1 & -1 & -1 & 0 & w_{41} & w_{42} & w_{43} & w_{44} & w_{45} \\
0 & -1 & 0 & 1 & 0 & w_{51} & w_{52} & w_{53} & w_{54} & w_{55} \\
0 & 0 & 0 & 0 & 0 & 0 & -1 & 0 & -1 & 0 \\
0 & 0 & 0 & 0 & 0 & 0 & 0 & 0 & 0 & 1 \\
0 & 0 & 0 & 0 & 0 & 1 & 0 & 0 & 0 & 0 \\
0 & 0 & 0 & 0 & 0 & 0 & 0 & -1 & 0 & -1 \\
0 & 0 & 0 & 0 & 0 & -1 & 0 & -1 & 1 & -1
\end{array}\right]
\end{aligned}
$$

Theorem 6.4. Let $S$ be a maximal symmetric Riemann surface of genus 5 admitting a maximal reflection $\tau: S \rightarrow S$. Let $p \in(2-\sqrt{3}, 1)$ be so that

(i) $S=\Omega / G_{5}$, if $S / \tau$ is genus one Riemann surface with 5 holes; or

(ii) $S=\Omega / F_{5}$, if $S / \tau$ is a genus zero surface with 6 holes. 
$A$ Riemann matrix of $S$ is given by $Z=P+i Q$, where $P=0$ in case (ii),

$$
\begin{aligned}
P & =\frac{1}{2}\left[\begin{array}{lllll}
0 & 1 & 1 & 0 & 0 \\
1 & 0 & 0 & 0 & 1 \\
1 & 0 & 0 & 0 & 1 \\
0 & 0 & 0 & 0 & 0 \\
0 & 1 & 1 & 0 & 0
\end{array}\right] \text { in case (i) } \\
Q & =\left[\begin{array}{ccccc}
2 u+v & u+v & u+v & v & 2(u+v) \\
u+v & 2 u+v & 2 u+3 v & -v & u+2 v \\
u+v & 2 u+3 v & 2 u+v & v & u \\
v & -v & v & -3 v & 2 v \\
2(u+v) & u+2 v & u & 2 v & 2 u
\end{array}\right],
\end{aligned}
$$

where $v=\frac{w}{2}-u$,

$$
w=\left\{\begin{array}{l}
\frac{1}{2 \pi} \log \left(\prod_{\gamma \in G_{2}} \frac{\left|\gamma\left(q_{2}\right)-A_{1}^{-1}(\infty)\right|}{\left|\gamma\left(q_{1}\right)-A_{1}^{-1}(\infty)\right|}\right) \quad \text { in case }(i) \\
\frac{1}{2 \pi} \log \left(\prod_{\gamma \in F_{2}} \frac{\left|\gamma\left(p_{2}\right)-B_{1}^{-1}(\infty)\right|}{\left|\gamma\left(q_{1}\right)-B_{1}^{-1}(\infty)\right|}\right) \quad \text { in case }(i i)
\end{array}\right.
$$

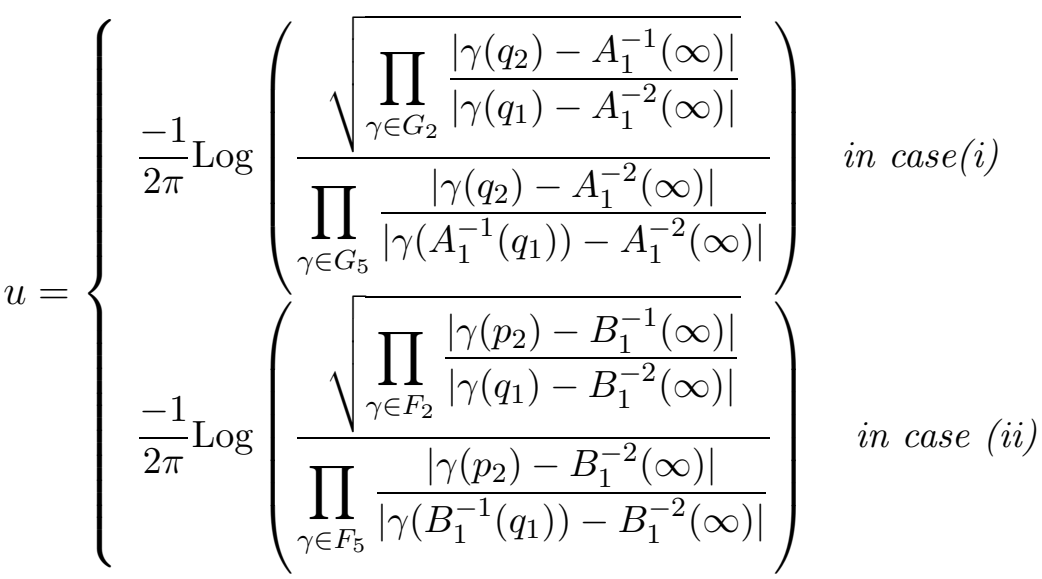

$\left.q_{1}=\exp (2 \pi / 3-\theta) i\right), q_{2}=J\left(q_{1}\right), p_{2}=\sigma_{p}\left(q_{1}\right)$ and $\theta \in\left(0, \frac{\pi}{2}\right)$ is such that $\cos \theta=2 p /\left(1+p^{2}\right)$.

Proof. Let us start recalling that the group $G_{5}$ (respectively, $F_{5}$ ) is a normal subgroup of the group $G_{2}$ (respectively, $F_{2}$ ), for each value of $p$. The surface uniformized by $G_{5}$ (respectively, $F_{5}$ ) is denoted by $S_{5}$ (respectively, $R_{5}$ ) and the surface uniformized by $G_{2}$ (respectively, $F_{2}$ ) is denoted by $S_{2}$ (respectively, $R_{2}$ ). We also have regular (unbranched) covering $P: S_{5} \rightarrow S_{2}$ (respectively, $P: R_{5} \rightarrow R_{2}$ ), whose covering group is the Klein group $\mathbb{Z}_{2}+\mathbb{Z}_{2}$ generated by the automorphisms of $S_{5}$ (respectively, $R_{5}$ ) defined by $A_{1}$ and $A_{2}$ (respectively, $B_{1}$ and $B_{2}$ ). 
The case $G_{5}$ : The Riemann matrix $Z=X+i Y \in \mathcal{H}_{5}$ of $S_{5}$ defined by the above adapted symplectic homology basis is a fixed point of the symplectic group $\rho\left(\widehat{K} / G_{5}\right)$. It follows that: $t_{n m}=j_{n m}=0, w_{11}=w_{15}=-w_{21}=$ $-w_{22}=-w_{31}=-w_{32}=w_{41}=w_{45}=-1, w_{22}=w_{23}=w_{24}=w_{31}=w_{24}=$ $w_{25}=w_{31}=w_{34}=w_{35}=w_{41}=w_{42}=w_{43}=w_{44}=w_{45}=w_{52}=w_{53}=$ $w_{54}=0$, and

$$
Z=\frac{1}{2}\left[\begin{array}{ccccc}
0 & 1 & 1 & 0 & 0 \\
1 & 0 & 0 & 0 & 1 \\
1 & 0 & 0 & 0 & 1 \\
0 & 0 & 0 & 0 & 0 \\
0 & 1 & 1 & 0 & 0
\end{array}\right]+i\left[\begin{array}{ccccc}
2 u+v & u+v & u+v & v & 2(u+v) \\
u+v & 2 u+v & 2 u+3 v & -v & u+2 v \\
u+v & 2 u+3 v & 2 u+v & v & u \\
v & -v & v & -3 v & 2 v \\
2(u+v) & u+2 v & u & 2 v & 2 u
\end{array}\right]
$$

where $0<-v<u$.

The case $F_{5}$ : The Riemann matrix $Z=X+i Y \in \mathcal{H}_{5}$ of $R_{5}$ defined by the above symplectic basis is a fixed point of the symplectic group $\eta\left(\widehat{K} / F_{5}\right)$. It follows that: $t_{n m}=j_{n m}=w_{n m}=0$, and

$$
Z=i\left[\begin{array}{ccccc}
2 u+v & u+v & u+v & v & 2(u+v) \\
u+v & 2 u+v & 2 u+3 v & -v & u+2 v \\
u+v & 2 u+3 v & 2 u+v & v & u \\
v & -v & v & -3 v & 2 v \\
2(u+v) & u+2 v & u & 2 v & 2 u
\end{array}\right]
$$

where $0<-v<u$.

\section{The relations between $\boldsymbol{w}, \boldsymbol{u}$ and $\boldsymbol{v}$}

Let us now consider the regular coverings $P: S_{5} \rightarrow S_{2}$ (respectively, $\left.P: R_{5} \rightarrow R_{2}\right)$. We have that $H_{1}(P): H_{1}\left(S_{5}, \mathbb{Z}\right) \rightarrow H_{1}\left(S_{2}, \mathbb{Z}\right)$ (respectively, $\left.H_{1}(P): H_{1}\left(R_{5}, \mathbb{Z}\right) \rightarrow H_{1}\left(R_{2}, \mathbb{Z}\right)\right)$ is given by $H_{1}(P)\left(\theta_{1}\right)=H_{1}(P)\left(\theta_{4}\right)=$ $H_{1}(P)\left(\theta_{5}\right)=\alpha_{1}, H_{1}(P)\left(\theta_{2}\right)=H_{1}(P)\left(\theta_{3}\right)=\alpha_{2}, H_{1}(P)\left(\delta_{1}\right)=H_{1}(P)\left(\delta_{5}\right)=$ $2 \beta_{1}, H_{1}(P)\left(\delta_{2}\right)=H_{1}(P)\left(\delta_{3}\right)=2 \beta_{2}$ and $H_{1}(P)\left(\delta_{4}\right)=0$. Let us denote by $w_{1}$ and $w_{2}$ the dual holomorphic one-forms of $\alpha_{1}$ and $\alpha_{2}$, respectively, for the surface $S_{2}$ (respectively, $R_{2}$ ). Similarly, let us denote by $\eta_{1}, \eta_{2}, \eta_{3}, \eta_{4}$ and $\eta_{5}$ the dual holomorphic one-forms of $\theta_{1}, \theta_{2}, \theta_{3}, \theta_{3}$ and $\theta_{5}$, respectively, for the surface $S_{5}$ (respectively, $R_{5}$ ). It follows then the pull-back of holomorphic forms $P^{*}: H^{1,0}\left(S_{2}\right) \rightarrow H^{1,0}\left(S_{5}\right)$ (respectively, $P^{*}: H^{1,0}\left(R_{2}\right) \rightarrow H^{1,0}\left(R_{5}\right)$ ) is defined by $P^{*}\left(w_{1}\right)=\eta_{1}+\eta_{4}+\eta_{5}$ and $P^{*}\left(w_{2}\right)=\eta_{2}+\eta_{3}$. Using the equality

$$
4(u+v) i=\int_{\delta_{1}} \eta_{1}+\eta_{4}+\eta_{5}=\int_{\delta_{1}} P^{*} w_{1}=2 \int_{\beta_{1}} w_{1}=2 i w,
$$

we obtain that $w=2(u+v)$. 
Again using the results due to Burnside [3] described at the end of section 3 to the groups $G_{5}$ and $F_{5}$ we obtain

$$
\begin{aligned}
& 2 u+v=-i \int_{\delta_{1}} \eta_{1}=\frac{1}{2 \pi} \log \left(\prod_{\gamma \in G_{5}} \frac{\left|\gamma\left(q_{2}\right)-A_{1}^{-2}(\infty)\right|}{\left|\gamma\left(A_{1}^{-1}\left(q_{1}\right)\right)-A_{1}^{-2}(\infty)\right|}\right), \quad \text { for } G_{5} \\
& 2 u+v=-i \int_{\delta_{1}} \eta_{1}=\frac{1}{2 \pi} \log \left(\prod_{\gamma \in F_{5}} \frac{\left|\gamma\left(q_{2}\right)-B_{1}^{-2}(\infty)\right|}{\left|\gamma\left(B_{1}^{-1}\left(q_{1}\right)\right)-B_{1}^{-2}(\infty)\right|}\right), \quad \text { for } F_{5} .
\end{aligned}
$$

From the above two equalities we obtain the forms for $u$ and $v$ as desired.

\section{Aknowledgments}

The author is very thankful to the referee for his/her valuable suggestions and questions. One of the referee's questions was the following: Given $p \in(2-\sqrt{3}, 1)$ we have the Schottky groups of genus two $G_{2}$ and $F_{2}$. To them we have a value of $\lambda=\lambda_{p}>1$ and a value of $\eta=\eta_{p} \in(0, \pi / 3)$ for the corresponding algebraic curves of 4.1. Is it possible to find a relation between $\lambda$ and $\eta$ ? For instance, for the value of $p$ obtained in 6.3. we have that $\eta=e^{\pi i / 6}$. What is the value of $\lambda$ ?

A partial answer to this question is given in 6.2. In there, for each value of $p$ we have the respective holomorphic map

$$
F=\frac{w_{1}}{w_{2}}
$$

for either case $G_{2}$ and $F_{2}$.

In the case of $F_{2}$ we have that $F(0)$ is the center of a circle containing the points $F\left(e^{-i \theta}\right), F(p)$ and $F\left(e^{i \theta}\right)$ in counterclockwise order. In this case we have that

$$
e^{i \eta_{p}}=\frac{F\left(e^{i \theta}\right)-F(0)}{F(p)-F(0)}
$$

In the case of $G_{2}$ we have that $F(0)$ is the center of three concentric circles, one of them, say the internal one, containing $F(p)$, the external one containing $F(1 / p)$ and the center one containing the point $F\left(e^{i \theta}\right)$. In this case we have that

$$
\lambda_{p}=\frac{F(1 / p)-F(0)}{F\left(e^{i \theta}\right)-F(0)}
$$

Of course the above does not give an answer to the above question, but it permits numerically to give the values of $\lambda_{p}$ and $\eta_{p}$ for the same value of $p$. 


\section{References}

[1] Bers, L.: Automorphic forms for Schottky groups. Advances in Math. 16 (1975), 332-361.

[2] Bujalance, E. and Etayo, J.J.: Hyperelliptic Klein surfaces with maximal symmetry. In Low-Dimensional topology and Kleinian groups (Coventry/Durham, 1984), 289-296. London Math. Soc. Lecture Notes Ser. 112. Cambridge Univ. Press, Cambridge, 1986.

[3] Burnside, W.: On a class of automorphic functions. Proc. London Math. Soc. 23 (1892), 49-88.

[4] Buser, P. and Silhol, R.: Geodesics, periods and equations of real hyperelliptic curves. Duke Math. J. 108 (2001), no. 2, 211-250.

[5] Chuckrow, V.: On Schottky groups with applications to kleinian groups. Ann. of Math. (2) 88 (1968), 47-61.

[6] Farkas, H. And Kra, I.: Riemann surfaces. Graduate Texts in Mathematics 71. Springer-Verlag, New York-Berlin, 1980.

[7] Gianni, P., Seppälä, M., Silhol, R. and Trager, B.: Riemann surfaces, plane algebraic curves and their period matrices. Symbolic numeric algebra for polynomials. J. Symbolic Comput. 26 (1998), no. 6, 789-803.

[8] Greenleaf, N. And May, C.L.: Bordered Klein surfaces with maximal symmetry. Trans. Amer. Math. Soc. 274 (1982), 265-283.

[9] Hidalgo, R.A.: On Schottky groups with automorphisms. Ann. Acad. Sci. Fenn. Ser. A I Math. 19 (1994), 259-289.

[10] Hidalgo, R.A.: Schottky uniformizations of closed Riemann surfaces with abelian groups of conformal automorphisms. Glasgow Math. J. 36 (1994), 17-32.

[11] Hidalgo, R.A.: Dihedral groups are of Schottky type. Proyecciones 18 (1999), 23-48.

[12] Hidalgo, R.A.: $\mathcal{A}_{4}, \mathcal{A}_{5}, \mathcal{S}_{4}$ and $\mathcal{S}_{5}$ of Schottky type. Rev. Mat. Complut. 15 (2002), no. 1, 11-29.

[13] Keen, L.: On hyperelliptic Schottky groups. Ann. Acad. Sci. Fenn. Ser. A I Math. 5 (1980), 165-174.

[14] Marden, A.: Schottky groups and circles. In Contributions to analysis (a collection of papers dedicated to Lipman Bers), 273-278. Academic Press, New York, 1974.

[15] Maskit, B.: Kleinian Groups. Grundlehren der Mathematischen Wissenschaften 287. Springer-Verlag, Berlin, 1988.

[16] Maskit, B.: Remarks on $m$-symmetric Riemann surfaces. In Lipa's legacy (New York, 1995), 433-445. Contemp. Math. 211. Amer. Math. Soc., Providence, RI, 1997.

[17] Maskit, B.: A characterization of Schottky groups. J. Analyse Math. 19 (1967), 227-230. 
[18] May, C. L.: Automorphisms of compact Klein surfaces with boundary. Pacific J. Math. 59 (1975), 199-210.

[19] MaY, C. L.: A bound for the number of automorphisms of a compact Klein surface with boundary. Proc. Amer. Math. Soc. 63 (1977), 273-280.

[20] May, C. L.: The species of bordered Klein surfaces with maximal symmetry of low genus. Pacific J. Math. 111 (1984), 371-394.

[21] Rodríguez, R.E. And GonzÁlez-Aguilera, V.: Fermat's quartic curve, Klein's curve and the tetrahedron. In Extremal Riemann surfaces (San Francisco, CA, 1995), 43-62. Contemp. Math. 201. Amer. Math. Soc., Providence, RI, 1997.

[22] SeppäLё, M.: Computation of period matrices of real algebraic curves. Discrete Comput. Geom. 11 (1994), 65-81.

[23] Seppälä, M. And Silhol, R.: Moduli spaces of for real algebraic curves and real abelian varieties. Math. Z. 201 (1989), 151-165.

[24] Vermeulen, L.: Non-hyperelliptic curves of genus three with Weierstrass points of weight two. Report 81-01, Department of Math. University of Amsterdam, 1981.

Recibido: 6 de junio de 2002

Rubén A. Hidalgo Departamento de Matemáticas Universidad Técnica Federico Santa María

Valparaíso, Chile ruben.hidalgo@mat.utfsm.cl 